\title{
Diversity Comparison of Pareto Front Approximations in Many-Objective Optimization
}

\author{
Miqing Li, Shengxiang Yang Member, IEEE, and Xiaohui Liu
}

\begin{abstract}
Diversity assessment of Pareto front approximations is an important issue in the stochastic multiobjective optimization community. Most of the diversity indicators in the literature were designed to work for any number of objectives of Pareto front approximations in principle, but in practice many of these indicators are infeasible or not workable when the number of objectives is large. In this paper, we propose a Diversity Comparison Indicator (DCI) to assess the diversity of Pareto front approximations in many-objective optimization. DCI evaluates relative quality of different Pareto front approximations rather than provides an absolute measure of distribution for a single approximation. In DCI, all the concerned approximations are put into a grid environment so that there are some hyperboxes containing one or more solutions. The proposed indicator only considers the contribution of different approximations to nonempty hyperboxes. Therefore, the computational cost does not increase exponentially with the number of objectives. In fact, the implementation of DCI is of quadratic time complexity, which is fully independent of the number of divisions used in grid. Systematic experiments are conducted using three groups of artificial Pareto front approximations and seven groups of real Pareto front approximations with different numbers of objectives to verify the effectiveness of DCI. Moreover, a comparison with two diversity indicators used widely in many-objective optimization is made analytically and empirically. Finally, a parametric investigation reveals interesting insights of the division number in grid and also offers some suggested settings to the users with different preferences.
\end{abstract}

Index Terms-Multiobjective optimization, many-objective optimization, performance assessment, diversity comparison indicator.

\section{INTRODUCTION}

$\mathbf{M}$ ANY real-world problems involve simultaneous optimization of several competing criteria or objectives: often, there is no single optimal solution, but rather a set of Pareto optimal solutions (also called Pareto front in the objective space). In general, generating the Pareto front is often infeasible, since the complexity of the underlying application prevents exact methods from being applicable. Heuristic search methods are an alternative: they try to find a good

Manuscript received September 5, 2013; revised December 29, 2013; accepted February, 26, 2014. This work was supported in part by the Engineering and Physical Sciences Research Council (EPSRC) of U.K. under Grant EP/K001310/1, National Natural Science Foundation of China (Major International Joint Research Project) under Grant 71110107026, EU FP7-Health under Grant 242193, EPSRC Industrial Case under Grant 11220252, and the Education Department and "Qinglan Engineering" of Jiangsu Province, China

M. Li and X. Liu are with the Department of Information Systems and Computing, Brunel University, Uxbridge, Middlesex UB8 3PH, U. K. (email: \{miqing.li, xiaohui.liu\}@ brunel.ac.uk).

S. Yang is with the Centre for Computational Intelligence (CCI), School of Computer Science and Informatics, De Montfort University, Leicester LE1 9BH, U. K. (e-mail: syang@dmu.ac.uk). approximation that is not too far away from the Pareto front, although they usually do not guarantee to obtain the optimal tradeoffs. Over the past decades, various stochastic heuristic search techniques, such as genetic algorithms, particle swarm optimization algorithms, ant colony optimization algorithms, simulated annealing, and tabu search, have been proposed to solve multiobjective optimization problems (MOPs), and their usefulness has been demonstrated in many application domains [1]-[5].

With the rapid development of useful and effective techniques in multiobjective optimization, the issue of performance assessment has become increasingly important and has developed into an independent research topic. Numerous quality indicators [6]-[10] have been emerging in the literature to evaluate the performance of Pareto front approximations obtained by multiobjective optimizers. They mainly concentrate on three aspects that the optimizers try their best to optimize: 1) the convergence of the obtained approximation, 2) the uniformity of the approximation, and 3) the spread (i.e., extensity) of the approximation. The latter two are closely related aspects, and in general, they are called the diversity of the approximation.

Many-objective optimization problems, which appear widely in industrial and engineering design [11]-[13], usually refer to those problems with more than three objectives. In recent years, many-objective optimization has been gaining increasing attention in the stochastic multiobjective optimization community: a great variety of many-objective optimizers have been developed [14]-[19], and their performance has been tested on many problems with different characteristics [20]-[25].

However, how to compare the Pareto front approximations obtained by different many-objective optimizers seems having not gotten enough attention and concern [26], which largely hinders the deep investigation of the performance of optimizers [27], [28]. Most of the quality indicators that are scalable analytically to any number of objectives are often not available actually to the problems with a large number of objectives. In general, the difficulties of comparing many-objective Pareto front approximations may be mainly attributed to three reasons, which are summarized as follows.

- Visual comparison of approximations. When the number of objectives of Pareto front approximations is more than three, visual and intuitive quality assessment can be misleading or even impossible, despite the fact that it is a prevailing comparison tool in the literature.

- Substitution of the Pareto front. Many quality indicators need to compare an approximation with a reference set, 
which is regarded as a substitution of the Pareto front. Due to the "curse of dimensionality", the number of points required to accurately represent the Pareto front of a given problem increases exponentially with the number of objectives. This places the setting of the number of points in a reference set in a dilemma: a large number will pose a challenge to the storage and access of data, whereas a small one will decrease the accuracy of the evaluation results.

- Complexity of storage and time. The storage or time requirement of some quality indicators, such as $h y$ pervolume [29], diversity measure [30], and hyperarea difference [31], increases exponentially with the number of objectives. This may limit their applicability in performance comparison of high-dimensional Pareto front approximations.

Whereas the above difficulties have created obstacles to the design of quality indicators in many-objective optimization, the influence for indicators on different aspects (i.e., convergence and diversity) may be of great difference. Some convergence indicators can avoid these difficulties by utilizing the characteristics of the Pareto front of the considered test problems [32], [33] or by testing the dominance relation between individuals of different approximations [9], [14], etc. However, for diversity indicators, a proper reflection of the distribution of approximations seems to be more difficult in many-objective optimization [7]. Separate assessment of uniformity and spread may give a misleading result about the whole distribution of an approximation (a detailed explanation will be given in the next section). In general, a diversity indicator without the introduction of comparison between an approximation and the problem's Pareto front fails to accurately reflect the distribution of the approximation, since the true shape and distribution of the optimal front are often unknown beforehand and hard to estimate in a high dimensional space.

In this paper, we propose a diversity ${ }^{1}$ comparison indicator (DCI) to compare Pareto front approximations in manyobjective optimization. DCI assesses the relative quality of different Pareto front approximations rather than provides an absolute measure of distribution for a single approximation, so that a reference set that is difficult to accurately represent the Pareto front of a many-objective optimization problem is not needed. Moreover, unlike binary quality indicators [34], [35], which compare the performance of two approximations, DCI is available for any number of approximations, which seems very attractive because it can identify all the considered approximations in a single run.

The rest of this paper is organized as follows. In Section II, the related work is reviewed and discussed. Section III is devoted to the description of the proposed performance indicator. Empirical comparative studies and discussions are presented in Section IV. Finally, Section V provides some concluding remarks along with pertinent observations.

\footnotetext{
${ }^{1}$ This paper focuses on the diversity in the objective space, rather than in the variable space, although the latter is also very important in the area.
}

\section{RELATED WORK}

During the past decade, various quality indicators have been emerging in the stochastic multiobjective optimization domain [7], [9], [36]. Some of them focus on the performance of Pareto front approximations in terms of a single aspect, and the others assess several aspects of the quality of approximations. Table I summarizes some quality indicators and their properties, listing the quality aspect(s) involved by the indicators, the number of approximations handled by the indicators, the computational effect needed by the indicators, and the state whether a reference set is required by the indicators or not.

As can be seen from Table I, there are three categories of existing quality indicators regarding the uniformity and spread of Pareto front approximations. The first category separately assesses the uniformity or spread of approximations (items 1118), the second one covers all three aspects of performance (i.e., uniformity and spread as well as convergence) (items 1924), and the last one focuses on diversity (i.e., both uniformity and spread, but no convergence) (items 25-27).

Considering the first category, although the indicators can separately evaluate the two aspects of diversity, they may fail to reflect the whole distribution of an approximation. Fig. 1 gives a distribution example to explain this case. The solutions of the approximation in the figure are uniformly distributed on the boundary of the Pareto front rather than over the whole Pareto front. In this case, both uniformity indicators (e.g., items 11-15) and spread indicators (e.g., items 1618) may give good evaluation results of the approximation. This occurrence can be attributed to the fact that the former only considers distribution uniformity in the neighborhood of solutions (e.g., the Spacing indicator [39] assesses the uniformity of an approximation by calculating the standard deviation of distance from each solution to its closest neighbor in the approximation), and the latter only measures the distribution range of boundary solutions rather than the coverage of an approximation to the whole Pareto front [60] (e.g., the Maximum Spread indicator [49] assesses the spread of an approximation by measuring the length of the diagonal of the minimal hyperbox that encloses the approximation). In fact, it may be meaningless that a uniformly-distributed solution set is located only in a small part of the Pareto front, which could be obtained more easily in many-objective optimization due to the exponential growth of the problem space [12], [26].

Note that for accuracy, in the remainder of the paper the meaning of "spread" is modified to refer to the performance of an approximation to cover the whole Pareto front.

The second category of indicators involve all three aspects of performance of approximations, and some of them, such as Hypervolume [29] and Inverted Generational Distance (IGD) [53], are popular to compare multiobjective optimizers in the literature [26], [61], [62]. However, there are some requirements for them to be used, which may obstruct their application in the performance comparison of approximations with a large number of objectives. Although Monte Carlo sampling-based approximate calculation can greatly reduce the computational cost of Hypervolume [63], the proper choice of the reference point in the calculation of the indicator is 
TABLE I

QUALITY INDICATORS AND THEIR PROPERTIES

\begin{tabular}{|c|c|c|c|c|c|c|c|}
\hline & Quality Indicators & Convergence & Uniformity & Spread & $\begin{array}{l}\text { Number of Ap- } \\
\text { proximations }\end{array}$ & Computational Effect & $\begin{array}{l}\text { Reference } \\
\text { Set Needed }\end{array}$ \\
\hline 1 & Generational Distance [37], $\mathrm{GD}_{p}[38]$ & $\sqrt{ }$ & & & unary & quadratic & $\sqrt{ }$ \\
\hline 2 & ONVG, GNVG [39]-[41] & $\sqrt{ }$ & & & unary & linear time & \\
\hline 3 & ONVGR, GNVGR [40], [41] & $\sqrt{ }$ & & & unary & linear time & $\sqrt{ }$ \\
\hline 4 & Convergence Measure [30] & $\sqrt{ }$ & & & unary & quadratic & $\sqrt{ }$ \\
\hline 5 & RNI [42] & $\sqrt{ }$ & & & unary & linear time & \\
\hline 6 & Error Ratio [40] & $\sqrt{ }$ & & & unary & quadratic & $\sqrt{ }$ \\
\hline 7 & $\mathrm{C} 1_{R}, \mathrm{C} 2_{R}[6],[43]$ & $\sqrt{ }$ & & & unary & quadratic & $\sqrt{ }$ \\
\hline 8 & Coverage [29], [44] & $\sqrt{ }$ & & & binary & quadratic & \\
\hline 9 & Dominance Ranking [8] & $\sqrt{ }$ & & & arbitrary & quadratic & \\
\hline 10 & Purity [45] & $\sqrt{ }$ & & & arbitrary & quadratic & \\
\hline 11 & Spacing [39], [46], Minimal Spacing [45] & & $\sqrt{ }$ & & unary & quadratic & \\
\hline 12 & Uniform Distribution [42] & & $\sqrt{ }$ & & unary & quadratic & \\
\hline 13 & Entropy Measure [47] & & $\sqrt{ }$ & & unary & linear time & \\
\hline 14 & Cluster [31] & & $\sqrt{ }$ & & unary & exponential in $m$ & \\
\hline 15 & Uniformity Assessment [48] & & $\sqrt{ }$ & & unary & quadratic & \\
\hline 16 & Maximum Spread [49]-[51] & & & $\sqrt{ }$ & unary & linear time & \\
\hline 17 & Overall Pareto Spread [31] & & & $\sqrt{ }$ & unary & linear time & \\
\hline 18 & Spread Assessment [52] & & & $\sqrt{ }$ & unary & exponential in $m$ & \\
\hline 19 & Hypervolume [29] & $\sqrt{ }$ & $\sqrt{ }$ & $\sqrt{ }$ & unary/binary & exponential in $m$ & \\
\hline 20 & Hyperarea Ratio [40] & $\sqrt{ }$ & $\sqrt{ }$ & $\sqrt{ }$ & unary & exponential in $m$ & $\sqrt{ }$ \\
\hline 21 & IGD [53], [54] $\operatorname{IGD}_{p}[38]$ & $\sqrt{ }$ & $\sqrt{ }$ & $\sqrt{ }$ & unary & quadratic & $\sqrt{ }$ \\
\hline 22 & Coverage Difference [55] & $\sqrt{ }$ & $\sqrt{ }$ & $\sqrt{ }$ & binary & exponential in $m$ & \\
\hline 23 & $G$-Metric [56], [57] & $\sqrt{ }$ & $\sqrt{ }$ & $\sqrt{ }$ & arbitrary & quadratic & \\
\hline 24 & Averaged Hausdorff Distance $\Delta_{p}$ [38] & $\sqrt{ }$ & $\sqrt{ }$ & $\sqrt{ }$ & unary & quadratic & $\sqrt{ }$ \\
\hline 25 & $\Delta$ Metric [58] & & $\sqrt{ }$ & $\sqrt{ }$ & unary & quadratic & \\
\hline 26 & Sigma Diversity Metric [59] & & $\sqrt{ }$ & $\sqrt{ }$ & unary & linear time & \\
\hline 27 & Diversity Measure [30] & & $\sqrt{ }$ & $\sqrt{ }$ & unary & exponential in $m$ & $\sqrt{ }$ \\
\hline 28 & The Proposed Indicator & & $\sqrt{ }$ & $\sqrt{ }$ & arbitrary & quadratic & \\
\hline
\end{tabular}

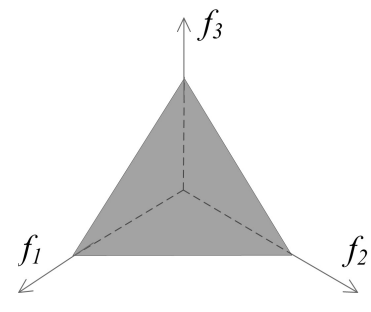

(a) Pareto front

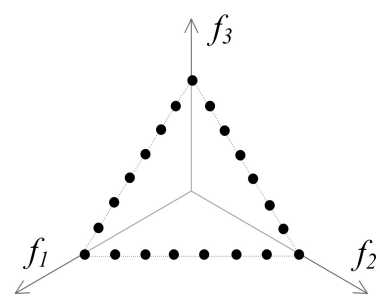

(b) An approximation
Fig. 1. A distribution example that uniformity or spread indicators may fail to reflect the whole distribution of an approximation.

also an important issue [64]. For IGD, a reference set that can accurately represent the Pareto front of a problem is required. Moreover, since these indicators concentrate on an overall consideration of convergence and diversity, they fail to separately reflect the distribution of approximations, which the user may be rather desirous to concern sometimes [65].

In addition, it is interesting to note that the comprehensive performance indicator G-Metric [56] (item 23 in Table I), which measures the convergence and diversity of approximations according to the Pareto dominance relation and "zone of influence" of individuals respectively, could be placed into the third category since their solutions are often mutually nondominated in a high dimension space. However, since the calculation of "zone of influence" is based on Monte Carlo sampling when more than three objectives are involved, the accuracy of the indicator may be affected as the number of objectives increases.

Represented by $\Delta$ Metric [58], Sigma Diversity Metric
(SDM) [59] and Diversity Measure (DM) [30], the third category of indicators assess the performance of approximations in terms of uniformity and spread. The $\Delta$ Metric is an extension of the uniformity indicator Spacing [39] by considering boundary solutions of the Pareto front for a biobjective problem, and it is defined as follows:

$$
\Delta=\frac{d_{f}+d_{l}+\sum_{i=1}^{|P|-1}\left|d_{i}-\bar{d}\right|}{d_{f}+d_{l}+(|P|-1) \bar{d}}
$$

where $|P|$ denotes the size of the considered approximation $P$, $d_{i}$ corresponds to the Euclidian distance between consecutive solutions in $P, \bar{d}$ stands for the average of $d_{i}$, and $d_{f}$ and $d_{l}$ denote the Euclidian distance between the extreme solution of the Pareto front and the boundary solution in the approximation regarding each of the two objectives, respectively. Due to the introduction of comparison between an approximation and the concerned Pareto front, the $\Delta$ metric seems to be one that reflects the distribution correctly. Despite the fact that $\Delta$ metric can easily be extended in a higher-dimensional space according to the Voronoi diagram approach, how to find the Voronoi diagram of a solution set is not an easy (or even an infeasible) task when more than three objectives are involved.

Inspired from the polar and spherical coordinate axis for the 2- and 3-objective space, the SDM indicator assesses the diversity of an approximation by using a set of reference lines dividing the objective space [59]. The outputs of the metric are a percentage of the space and the position information of a given approximation in the space, rather than a scalar value representing the distribution of the approximation. However, due to depending on several parameters, such as the distance 
around each reference line, the number of reference lines, and the shape of the Pareto front, the metric may face difficulties to be extended to many-objective optimization.

DM, proposed by Deb at el. [30], has recently been used in some comparison studies of many-objective optimizers [32], [66], [67]. DM measures the diversity of a Pareto front approximation by comparing it with a reference set. In the calculation of DM, the solutions in the approximation are projected on a $(m-1)$-dimensional hyperplane which is divided into a number of hyperboxes. The indicator considers each hyperbox and gives it an evaluation value according to the distribution of solutions in it and its neighbors. The more the hyperboxes that, when containing a member of the reference set, also contain a member of the approximation, the higher the indicator value. DM takes the value between zero and one, where one corresponds to the best possible diversity and zero stands for the worst. A more detailed description of DM can be found in [30].

The idea of assessing diversity using a grid-based way is meaningful, but DM has some shortcomings when applied to compare Pareto front approximations with a large number of objectives, which are described as follows:

- A reference set, in which the solutions are uniformly distributed over the Pareto front, is required in order to accurately reflect the distribution of the optimal front; and it is also required that the number of solutions in the reference set is approximate to the number of solutions in the approximation in order to guarantee that the ideal distribution of the approximation can reach the optimal $\mathrm{DM}$ value (one). These requirements are often unavailable in many-objective optimization problems.

- DM needs to access each hyperbox in grid to estimate the distribution, which produces great challenges to both the data structure and computational cost. For an optimization problem with $m$ objectives, there will be $r^{m-1}$ hyperboxes to be considered, where $r$ is the number of divisions in each dimension.

- In the distribution estimation for a hyperbox, DM needs to assign each of its neighboring hyperboxes a proper value by a value function to distinguish different distributions of solutions in its neighborhood. Since the number of neighbors of a hyperbox increases exponentially with the number of objectives (there are $\left(3^{m}-1\right)$ neighbors for an $m$-dimensional hyperbox at most), the value function accurately reflecting different distributions will be difficult to define when the concerned problem involves a large number of objectives [30].

- DM may fail to give an accurate diversity result of an approximation with a large number of objectives due to the designation of the neighbors of a solution in grid. The setting of neighborhood of a solution in DM is based on the Manhattan distance of grid coordinates of solutions, rather than on the Euclidean distance of them, which may misleadingly eliminate adjacent solutions but regard farther ones as its neighbors (a detailed analysis will be given in Section IV-C).

The diversity indicator proposed in this paper also adopts a grid-based technique to compare the distribution of Pareto front approximations, but it attempts to solve all the above difficulties. Some of its properties are also shown in Table I. A key difference between DCI and DM lies in that DCI only considers the hyperboxes where the nondominated solutions in approximations are located, so that at most $N$ hyperboxes will be accessed for an approximation, where $N$ is the size of the approximation. The proposed DCI has a quadratic computational complexity, which is not affected by the number of divisions in grid, and thus can be easily calculated to examine and compare the diversity of approximations for many-objective optimization problems.

\section{ThE DIVERSITY COMPARISON INDICATOR (DCI)}

The essential idea behind the proposed indicator is to consider the contribution of different Pareto front approximations to the hyperboxes that have at least one nondominated solution. All the concerned approximations are put into a grid environment so that there are some hyperboxes containing one or more nondominated solutions. Depending on the contribution of an approximation to these hyperboxes, the diversity indicator of the approximation is defined. If the contributions of an approximation to all of these hyperboxes are maximal, the best diversity value is achieved; if the contributions of an approximation to most of these hyperboxes are low, the diversity is poor. Below, we introduce the grid environment where the DCI is implemented, followed by the formulation of the DCI and its time requirement analysis.

\section{A. Grid Environment}

The position and size of grid are of great importance in the proposed indicator. The grid should not involve the whole objective space but rather aim at a region not far away from the Pareto front of a given problem, because a prerequisite of meaningful diversity comparison of different approximations is that they have already approached the optimal front [50]. Assuming that the lower and upper boundaries of grid are $L B=\left(l b_{1}, l b_{2}, \ldots, l b_{m}\right)$ and $U B=\left(u b_{1}, u b_{2}, \ldots, u b_{m}\right)$ respectively ( $m$ denotes the number of objectives), a solution vector $\left(q_{1}, q_{2}, \ldots, q_{m}\right)$ that goes beyond $L B$ or $U B$ (i.e., $k \in\{1,2, \ldots, m\}: q_{k}<l b_{k}$ or $q_{k}>u b_{k}$ ) will be discarded in the indicator calculation.

In the application of the proposed DCI to different problems, the grid boundary may be determined by the "satisfied region" defined by the user, or be set by using the Ideal point and Nadir point of the problems ${ }^{2}$. The "satisfied region" is a user's estimation that the obtained solutions in it are considered to achieve the quality requirement in terms of convergence. When the user fails to clearly define his/her "satisfied region", the grid boundary can be obtained by the Ideal point and Nadir point of a given problem (shown in Fig. 2). The Ideal point and Nadir point are two important concepts in multiobjective optimization, and they can be estimated by some efficient methods when the boundary of the Pareto front is unknown [1],

\footnotetext{
${ }^{2}$ The Ideal point is an $m$-dimensional vector constructed with the best objective values, and the Nadir point signifies the opposite (i.e., constructed with the worst objective values of the Pareto front).
} 


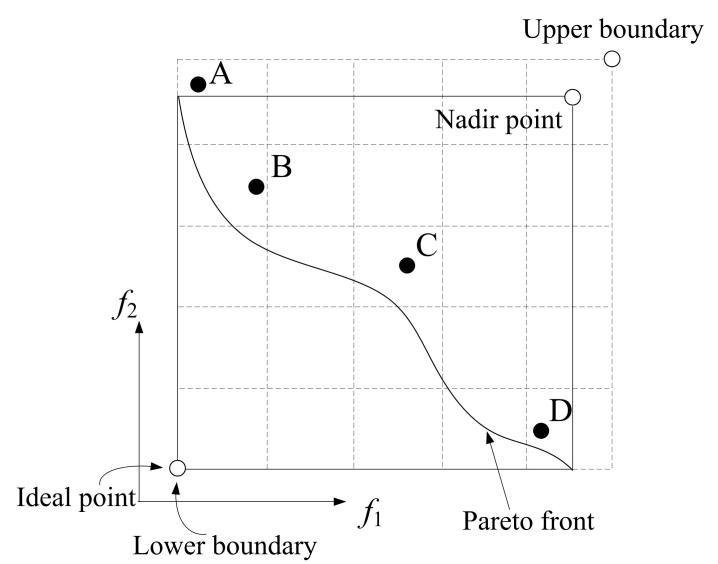

Fig. 2. Grid setting for a bi-objective minimization problem.

[68], [69]. Here, a slight relaxation of the region constructed by the Ideal point and Nadir point of a problem is regarded as the grid environment:

$$
\begin{gathered}
u b_{k}=n p_{k}+\frac{n p_{k}-i p_{k}}{2 \times d i v} \\
l b_{k}=i p_{k}
\end{gathered}
$$

where $i p_{k}$ and $n p_{k}$ denote the values of the Ideal point and Nadir point in the $k$ th objective, respectively, and div is a constant parameter, i.e., the number of divisions of the objective space in a dimension, set by the user (e.g., div $=5$ in Fig. 2).

According to the boundaries of grid and the number of divisions, the hyperbox size $d_{k}$ in the $k$ th objective can be formed as follows:

$$
d_{k}=\frac{u b_{k}-l b_{k}}{d i v}
$$

In this case, the grid location of a solution in Pareto front approximations can be determined by the lower boundary and the hyperbox size as follows:

$$
G_{k}(q)=\left\lfloor\left(F_{k}(q)-l b_{k}\right) / d_{k}\right\rfloor
$$

where $G_{k}(q)$ denotes the grid coordinate of solution $q$ in the $k$ th objective, and $F_{k}(q)$ is the actual objective value in the $k$ th objective. For example, the grid coordinates of solutions $\mathbf{A}, \mathbf{B}, \mathbf{C}$, and $\mathbf{D}$ in Fig. 2 are $(0,4),(0,3),(2,2)$, and $(4,0)$, respectively. In the following, several distance-based concepts used in the DCI calculation are introduced.

Definition 1 (Grid distance between two hyperboxes). Let $h_{1}$ and $h_{2}$ be two hyperboxes in grid, the grid distance between them is calculated as follows:

$$
G D\left(h_{1}, h_{2}\right)=\sqrt{\sum_{k=1}^{m}\left(h_{1}^{k}-h_{2}^{k}\right)^{2}}
$$

where $h_{1}^{k}$ and $h_{2}^{k}$ denote the coordinate of $h_{1}$ and $h_{2}$ in the $k$ th objective respectively, and $m$ is the number of objectives. For example, the grid distance between the hyperboxes where solutions $\mathbf{B}$ and $\mathbf{C}$ are located is $\sqrt{(0-2)^{2}+(3-2)^{2}}=\sqrt{5}$ in Fig. 2.

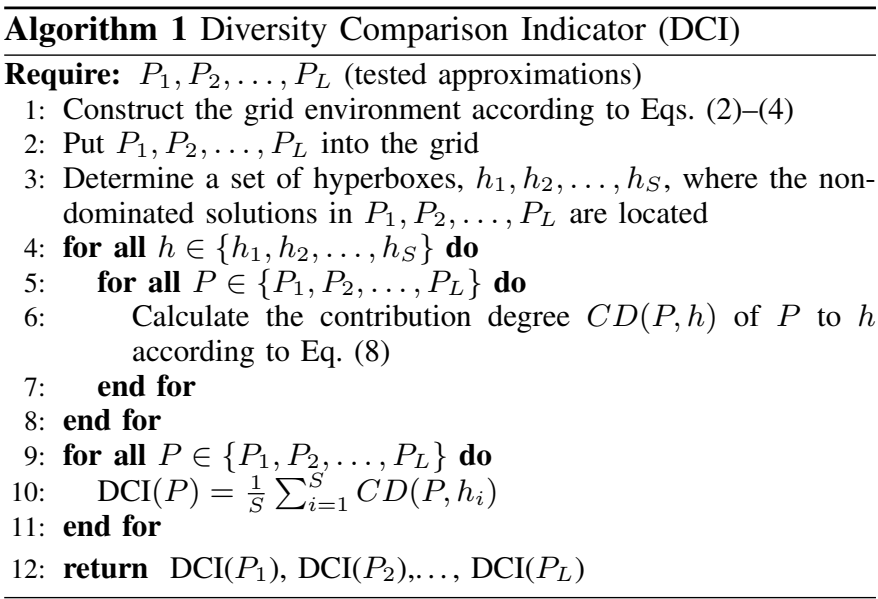

Definition 2 (Distance from an approximation to a hyperbox). Let $P$ be an approximation and $h$ a hyperbox in grid. The distance from $P$ to $h$ is the shortest grid distance between $h$ and the hyperbox that has at least one solution of $P$ :

$$
D(P, h)=\min _{p \in P}\{G D(h, G(p))\}
$$

where $G(p)$ denotes the hyperbox where solution $p$ is located. For example, in Fig. 2, the distance from the approximation composed of solutions $\mathbf{A}, \mathbf{B}, \mathbf{C}$, and $\mathbf{D}$ to the hyperbox of coordinates $(1,3)$ is equal to $G D\left(h^{(1,3)}, G(\mathbf{B})\right)=1$. Apparently, an approximation whose solutions are uniformly and extensively distributed in the grid environment has a low average distance value to all hyperboxes.

\section{B. Diversity Comparison of Approximations}

The solutions in different Pareto front approximations may be located in different hyperboxes. Here, we only consider the hyperboxes where the nondominated solutions in the mixed set of the considered approximations are located, because the diversity of dominated solutions may be meaningless for the user. For an approximation, if its solutions cover or are close to all of the considered hyperboxes, it will achieve a relatively good diversity in comparison with other approximations; on the other hand, if its solutions are far away from most of these hyperboxes, a relatively poor diversity will be obtained. Algorithm 1 gives the main procedure of calculating the DCI values for approximations being compared.

The contribution degree (line 6 of Algorithm 1) reflects the contribution of an approximation to a hyperbox and is determined by the distance between them. For an approximation, if there exists at least one solution in the considered hyperbox, the maximum contribution degree of the approximation to the hyperbox will be achieved; if the distance from the approximation to the hyperbox is farther than a specified threshold (i.e., the hyperbox's neighborhood), the contribution degree will be assigned zero. Specifically, the contribution degree of an approximation $P$ to a hyperbox $h$ is defined as:

$C D(P, h)= \begin{cases}1-D(P, h)^{2} /(m+1), & D(P, h)<\sqrt{m+1} \\ 0, & D(P, h) \geq \sqrt{m+1}\end{cases}$ 


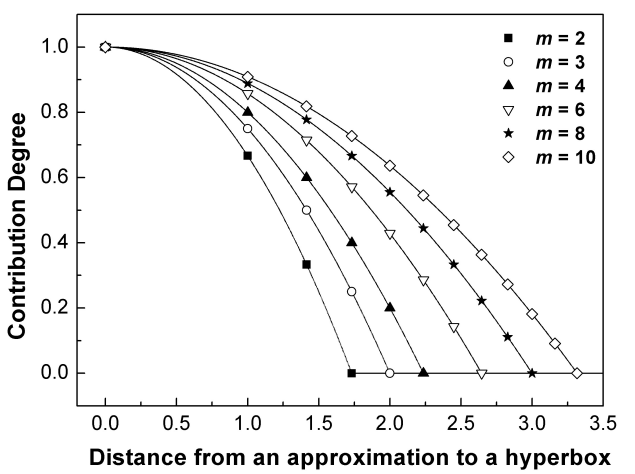

Fig. 3. The contribution degree function regarding different numbers of objectives.

where $D(P, h)$ denotes the distance from $P$ to $h$ as defined in Eq. (7), and $m$ denotes the number of objectives.

It is worth pointing out that we set the threshold of grid distance to $\sqrt{m+1}$ in order to ensure that two adjacent individuals can always interact (i.e., the hyperboxes where they are located are always neighbors to each other). Intuitively, two hyperboxes should be regarded as neighbors if their individuals can arbitrarily approach (i.e., there is no another hyperbox between the individuals). Clearly, the two farthest hyperboxes that meet the above condition are diagonal hyperboxes whose grid distance is $\sqrt{m}$. Since the grid distance between hyperboxes is always a discrete value $(\sqrt{0}, \sqrt{1}, \ldots, \sqrt{m}, \sqrt{m+1}, \ldots)$, setting the threshold to $\sqrt{m+1}$ just makes such diagonal hyperboxes to be neighbors and thus to be able to interact with each other in the calculation of the contribution degree.

Fig. 3 shows the curves of the contribution degree function regarding different numbers of objectives. Note that the contribution degree takes a discrete value since $D(P, h) \in$ $\{\sqrt{0}, \sqrt{1}, \ldots, \sqrt{m}, \sqrt{m+1}, \ldots\}$. From the figure, some observations can be drawn as follows.

- The contribution degree takes the value between zero and one. It decreases monotonously with the increase of the distance from an approximation to a hyperbox in a certain range (i.e., the neighborhood of the hyperbox).

- The radius of a hyperbox's neighborhood increases with the number of objectives. This indicates that a larger range can be considered for individuals to interact when the number of objectives becomes higher.

- For equal values of the distance variable $D(P, h)$, the contribution degree increases with the number of objectives. This increase seems reasonable since the relative distance between hyperboxes becomes smaller with the growth of the total number of hyperboxes in the grid.

Overall, the contribution degree function considers not only the distance information from an approximation to a hyperbox but also the properties of the grid environment with different numbers of objectives, thereby showing a good adaptability to the change of the number of objectives. In fact, any form of function can be assigned as a contribution degree function by keeping in mind the above properties. Here, a quadratic function is used for simplicity.

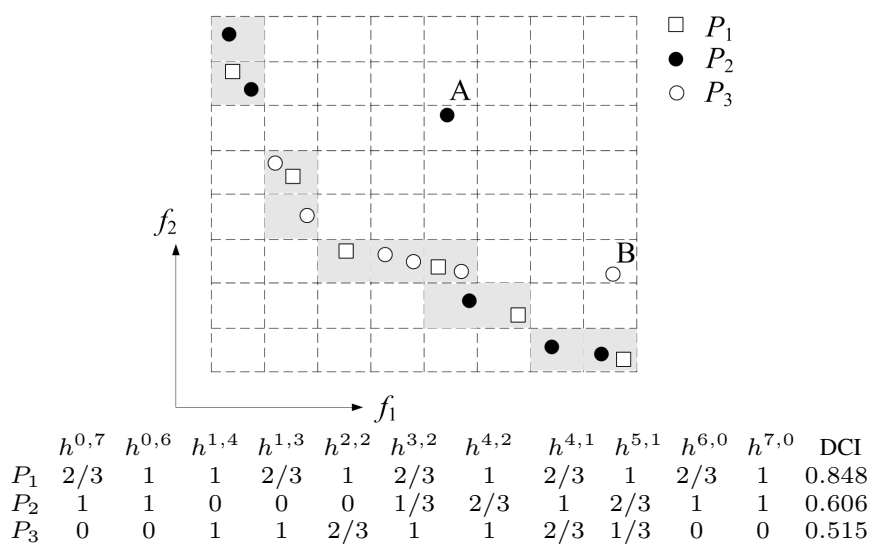

Fig. 4. An illustration of DCI calculation. The considered hyperbox $h^{i, j}$ is highlighted with a gray background, where $i$ and $j$ denote the coordinates in objectives $f_{1}$ and $f_{2}$, respectively. The number corresponding to an approximation $P$ and a hyperbox $h$ means the contribution degree of $P$ to $h$ (i.e., $C D(P, h)$ ).

According to the contribution degree function, the DCI value of an approximation is a number in the range $[0,1]$. It is necessary to reiterate that the DCI only assesses the relative distribution quality of different Pareto front approximations rather than provides an absolute measure of distribution for a single approximation. The best value (i.e., DCI $=1$ ) obtained by an approximation cannot reflect that it is uniformly distributed over the whole Pareto front. Instead, it indicates that the approximation has a perfect advantage over other approximations: it covers all the hyperboxes where the nondominated solutions belonging to other approximations are located. On the other hand, a well-distributed approximation may not reach the best DCI value if it fails to cover all the hyperboxes that the nondominated solutions belonging to other approximations occupy.

As an illustration to the calculation procedure of the DCI, Fig. 4 shows three Pareto front approximations $P_{1}, P_{2}$, and $P_{3}$ for a bi-objective problem. First, $P_{1}, P_{2}$, and $P_{3}$ are put into the grid environment, and then 11 hyperboxes (marked in gray) are determined (here, solutions A and B's hyperboxes are not considered since they are dominated solutions in the mixed set). Afterward, for each of these hyperboxes, the contribution degree of the three approximations is calculated according to Eq. (8). For example, considering hyperbox $h^{0,7}(0$ and 7 correspond to its coordinates in $f_{1}$ and $f_{2}$, respectively), the contribution degree of $P_{2}$ reaches 1 since $P_{2}$ has a solution in this hyperbox; the contribution degree of $P_{1}$ is $1-1^{2} / 3=2 / 3$ as $D\left(P_{1}, h^{0,7}\right)=1$; for approximation $P_{3}$, the contribution degree is equal to zero because the distance from $P_{3}$ to $h^{0,7}$ is farther than the threshold $\left(D\left(P_{3}, h^{0,7}\right)=\sqrt{10}>\sqrt{3}\right)$. Finally, the average contribution degree of each approximation to these hyperboxes is obtained according to Algorithm 1 (line 10), and the DCI values of $P_{1}, P_{2}$, and $P_{3}$ are $0.848,0.606$, and 0.515 , respectively.

\section{Time Requirement of DCI}

The computational cost of DCI can mainly be divided into three parts. The first involves the operations of calculating 


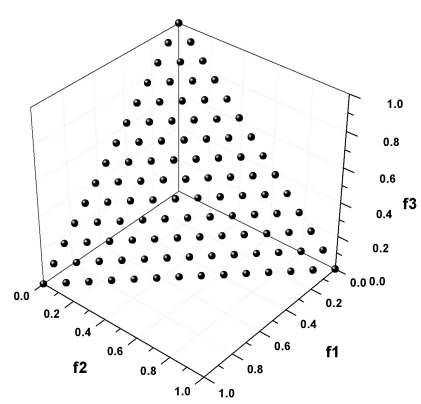

(a) $\mathrm{DCI}=0.8000$

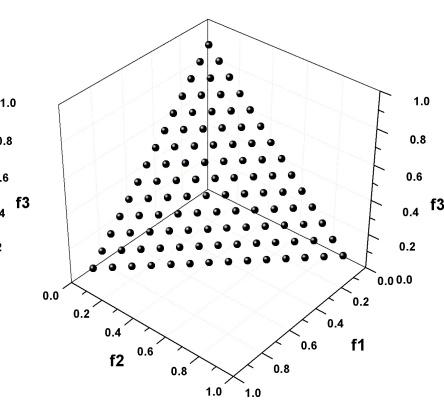

(b) $\mathrm{DCI}=0.7816$

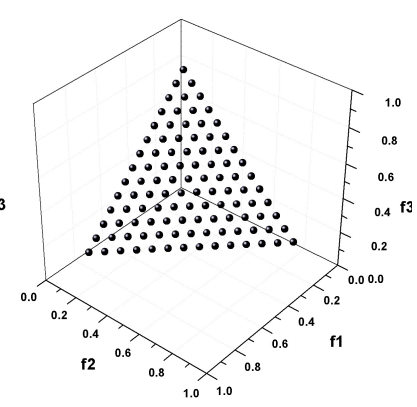

(c) $\mathrm{DCI}=0.7026$

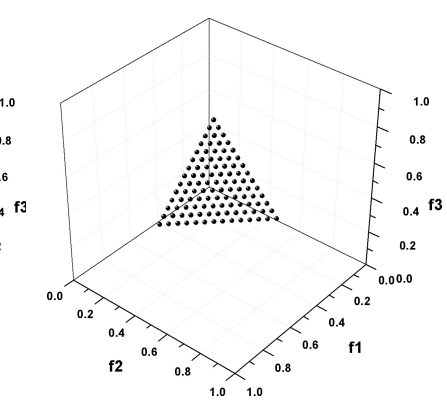

(d) DCI $=0.4026$

Fig. 5. DCI test for a group of artificial Pareto front approximations with different distribution ranges. All solutions in the approximations are located on the Pareto front $f_{1}+f_{2}+f_{3}=1$.

the grid coordinates for the solutions in all approximations, which requires $O(m L N)$ computations for an $m$-objective problem, where $L$ denotes the number of approximations and $N$ is the approximation size. The second part is related to the identification of nondominated solutions in the mixed set and to the record of their hyperboxes in grid, which requires $O\left(m(L N)^{2}\right)$ comparisons. Clearly, the number of the considered hyperboxes, denoted as $S$, is not larger than $L N$. The third part corresponds to the operation of calculating the contribution degree of all approximations to the $S$ hyperboxes. This requires $O(m L N S)$ computations since at most $m L N$ comparisons are implemented for one hyperbox.

From the above analysis, the total complexity of the DCI is $\max \left(O\left(m(L N)^{2}\right), O(m L N S)\right)=O\left(m(L N)^{2}\right)$, since $S \leq$ $L N$. This cost is fully determined by the number of objectives and the size summation of all approximations, and thus is independent of the grid division and does not increase with the number of hyperboxes.

\section{EXPERIMENTS AND DISCUSSIONS}

In this section, we verify the proposed DCI indicator. First, three groups of artificial Pareto front approximations are introduced to show the effectiveness of DCI in assessing the spread and uniformity of approximations. Then, seven groups of Pareto front approximations obtained by six multiobjective optimizers in different numbers of objectives are used to further examine DCI, in view of their representative results in terms of diversity. Next, a comparison with two diversity indicators used widely in many-objective optimization is made analytically and empirically. Finally, the effect of the parameter (i.e., the grid division) in DCI is investigated, and a further discussion of the proposed indicator is made.

\section{A. Artificial Examples}

As mentioned before, diversity of Pareto front approximations involves two facets, i.e., spread and uniformity. Here, we illustrate the validity of the proposed indicator by introducing several groups of artificial Pareto front approximations with different characteristics in spread and uniformity, with respect to a tri-objective optimization problem with a Pareto front $f_{1}+f_{2}+f_{3}=1\left(0 \leq f_{1}, f_{2}, f_{3} \leq 1\right)$.

Fig. 5 gives a group of artificial Pareto front approximations. Each one has 105 solutions distributed uniformly on the Pareto front of the problem. The only difference is their distribution range: the solutions in Fig. 5(a) are located over the whole Pareto front, and the solutions in other figures are located on a part of the Pareto front (from Fig. 5(b) to Fig. 5(d), the objective value of solutions belongs to the ranges [0.05, 0.9], [0.1,0.8], and [0.2, 0.6], respectively). Now, we apply the proposed indicator on these Pareto front approximations. The number of grid divisions is set to 19 for this and other tri-objective problems, unless otherwise stated. As can be seen from Fig. 5, the evaluation results given in the figure are consistent with the distribution range of the Pareto front approximations, which indicates that DCI can accurately reflect the spread of approximations.

The Pareto front approximations in Fig. 6 are used to test the DCI in terms of distribution uniformity. The uniformity degree of four approximations is decreased gradually from Fig. 6(a) to Fig. 6(d), although all of them are located over the whole Pareto front. Clearly, the evaluation results show the effectiveness of DCI in assessing a set of approximations with different uniformity degrees. In addition, note that the same approximation in Fig. 5(a) and Fig. 6(a) has different DCI results since it has different competitors in the diversity comparison.

The above two groups of Pareto front approximations verify the correctness of the DCI in evaluating spread and uniformity separately. However, a comprehensive evaluation on a group of Pareto front approximations with different distribution ranges and uniformity degrees is necessary to further test the DCI; e.g., for two approximations, how to compare their diversity, if one performs better in terms of spread and the other has an advantage in uniformity. Fig. 7 shows this case. A group of Pareto front approximations are formed by copying the approximations in Figs. 5 and 6 (i.e., Fig. 7(a) = Fig. 6(c), Fig. $7(\mathrm{~b})=$ Fig. 5(c), Fig. $7(\mathrm{c})=$ Fig. 6(d), and Fig. 7(d) $=$ Fig. 5(d)). From the evaluation results in the figure, DCI could be considered as a tradeoff evaluation between spread and uniformity. An approximation with a great advantage over its competitors at one point will achieve a better DCI value, even though it performs slightly worse at the other point.

\section{B. Real Examples}

In this section, we apply the proposed indicator on Pareto front approximations obtained by six established multiobjec- 


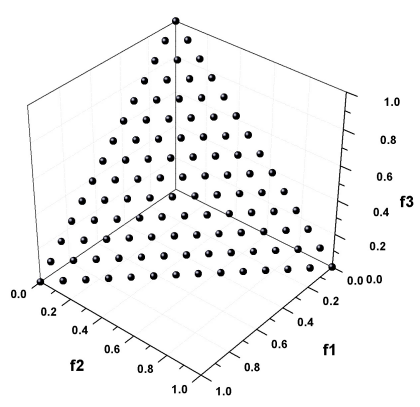

(a) $\mathrm{DCI}=0.8245$

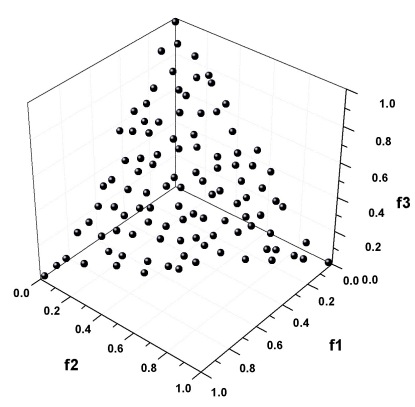

(b) $\mathrm{DCI}=0.7979$

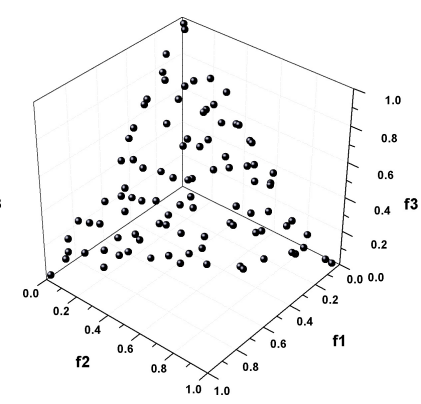

(c) $\mathrm{DCI}=0.7500$

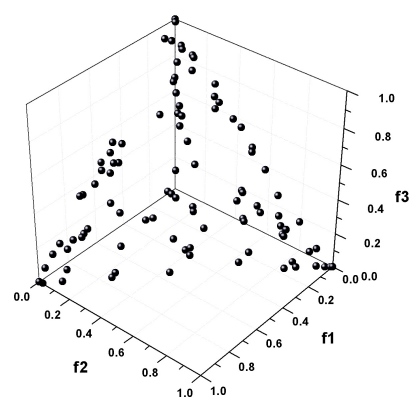

(d) $\mathrm{DCI}=0.6809$

Fig. 6. DCI test for a group of artificial Pareto front approximations with different uniformity degrees. All solutions in the approximations are located on the Pareto front $f_{1}+f_{2}+f_{3}=1$.

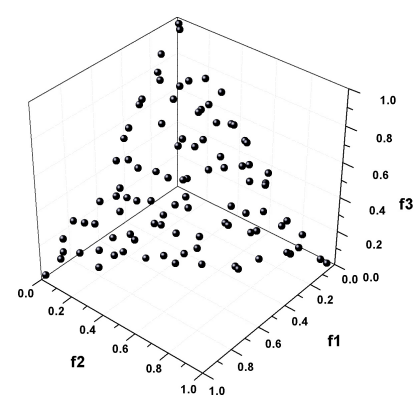

(a) $\mathrm{DCI}=0.7463$

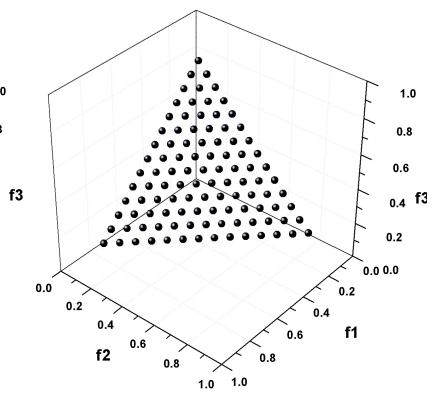

(b) $\mathrm{DCI}=0.6970$

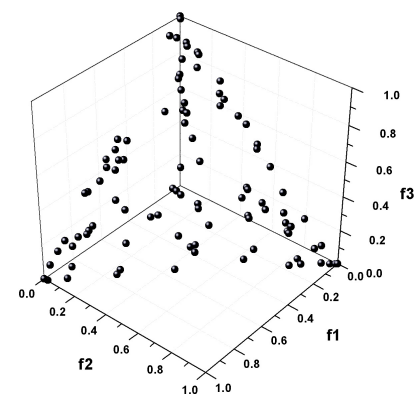

(c) $\mathrm{DCI}=0.6329$

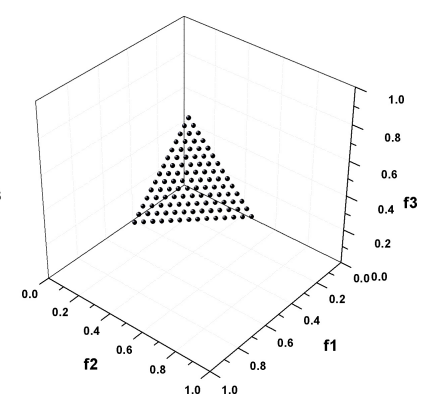

(d) $\mathrm{DCI}=0.4377$

Fig. 7. DCI test for a group of artificial Pareto front approximations with different distribution ranges and uniformity degrees. All solutions in the approximations are located on the Pareto front $f_{1}+f_{2}+f_{3}=1$.

tive optimizers, which are described as follows.

\section{- Nondominated Sorting Genetic Algorithm II (NSGA-}

II) [58]. This is one of the most popular evolutionary multiobjective optimization (EMO) algorithms in the literature. The main characteristics of NSGA-II are fast nondominated sorting and crowding distance-based density estimation in fitness assignment.

- Average Ranking (AR) [70]. AR is regarded as a good alternative to rank solutions in a multiobjective population. It compares all solutions in each objective and independently ranks them, and the final rank of a solution is obtained by summing its ranks of all objectives. AR is found to perform successfully in searching towards the optimal direction for many-objective optimization problems [14], [17], although often converging into a subset of the Pareto front due to the lack of diversity maintenance schemes [17], [67].

- Indicator-Based Evolutionary Algorithm (IBEA) [71]. IBEA, introduced by Zitzler and Küenzli, aims to integrate the preference information of the decision maker into the multiobjective search. The main idea is to first define the optimization goal in terms of a binary performance measure and then directly use this measure in the mating and environmental selection processes. Since the single measure that involves both convergence and diversity is used to optimize a desired property of an approximation, IBEA is competitive with classic EMO algorithms in many-objective optimization [33].

- Diversity Management Operator (DMO) [51]. DMO is a methodology to manage the use of diversity preservation operators in dealing with many-objective problems. It adaptively tunes the diversity operator according to the requirement of the evolutionary population. If the diversity result is smaller than 1 by the Maximum Spread test [49], a specified diversity promotion mechanism is activated; otherwise, deactivated. Specifically in [51], DMO is implemented in NSGA-II and the crowding distance is regarded as the diversity promotion mechanism.

- Territory Defining Evolutionary Algorithm (TDEA) [72]. TDEA is a steady-state algorithm based on the concept of territory. Keeping the Pareto dominance relation in mind, the algorithm defines a territory around each solution and forbids other solutions to reside in it, thereby providing a good tradeoff between convergence and diversity.

- Average Ranking combined with Grid (AR+Grid) [67]. AR+Grid is a hybrid method which uses grid to enhance diversity for AR in many-objective optimization. In AR+Grid, the AR strategy is employed to provide the selection pressure searching towards the Pareto front, and grid instrument is introduced to prevent solutions from being crowded in the objective space.

All tested multiobjective optimizers are given real-valued decision variables. A crossover probability $p_{c}=1.0$ and a mutation probability $p_{m}=1 / l$ (where $l$ is the number of decision variables) are used. Simulated binary crossover (SBX) and polynomial mutation are separately chosen as crossover and mutation operators [3]. Both of them use the distribution 
TABLE II

SETTINGS OF PARAMETERS $\tau$ AND $d$ IN TDEA AND AR+GRID

\begin{tabular}{c|cccccc}
\hline Number of Objectives & 3 & 4 & 5 & 6 & 8 & 10 \\
\hline$\tau$ & 0.10 & 0.22 & 0.34 & 0.44 & 0.67 & 0.86 \\
$d$ & 20 & 17 & 15 & 13 & 13 & 13
\end{tabular}

TABLE III

PARAMETER SETTINGS OF DCI

\begin{tabular}{c|cccccc}
\hline Number of Objectives & 3 & 4 & 5 & 6 & 8 & 10 \\
\hline div & 19 & 11 & 10 & 8 & 6 & 5
\end{tabular}

TABLE IV

DCI RESULTS OF THE SIX OPTIMIZERS ON DTLZ2 WITH DIFFERENT NUMBERS OF OBJECTIVES

\begin{tabular}{c|c|c|c|c|c|c}
\hline Algorithm & 3 obj. & 4 obj. & 5 obj. & 6 obj. & 8 obj. & 10 obj. \\
\hline NSGA-II & 0.5777 & 0.5365 & 0.0684 & 0.0000 & 0.0000 & 0.0000 \\
AR & 0.1841 & 0.0210 & 0.0172 & 0.0229 & 0.0420 & 0.0545 \\
IBEA & 0.3843 & 0.4096 & 0.4301 & 0.5132 & 0.2811 & 0.3381 \\
DMO & 0.5455 & 0.5178 & 0.4240 & 0.3942 & 0.4515 & 0.4266 \\
TDEA & 0.7365 & 0.6901 & 0.5957 & 0.5983 & 0.4862 & 0.4878 \\
AR+Grid & 0.6927 & 0.6436 & 0.5922 & 0.6391 & 0.7066 & 0.7590 \\
\hline
\end{tabular}

indexes 20 (i.e., $\eta_{c}=20$ and $\eta_{m}=20$ ). For each optimizer, a population ${ }^{3}$ of 100 individuals and a predefined number of 30,000 evaluations are set. Additionally, for TDEA and AR+Grid, two parameters $\tau$ and $d$ (i.e., the size of territory in TDEA and the number of grid divisions in AR+Grid) are required respectively. They are given in Table II.

To verify the proposed indicator, two scalable test function DTLZ2 and DTLZ7 [20] are used. The Pareto front of DTLZ2 corresponds to the positive part of the unit hypersphere, and the Pareto front of DTLZ7 consists of $2^{m-1}$ disconnected regions. The total number of decision variables in the functions is $l=m+n-1$, where $m$ denotes the number of objectives and $n$, set by users, is a parameter specifying the distance from solutions to the Pareto front. According to [20], here $n$ is set to 10 and 20 for DTLZ2 and DTLZ7, respectively.

In the proposed DCI, a parameter div (i.e., the number of grid divisions) is required to divide the considered region into many hyperboxes. In this section, the setting of div is fixed and shown in Table III. A detailed investigation about different configurations of div will be given in Section IV-D.

Table IV gives the diversity comparison results of the Pareto front approximations obtained by the six optimizers for different numbers $(3,4,5,6,8$, and 10) of objectives of DTLZ2. Due to the space limitation, only the distributions of the approximations for 3-, 6-, and 10-objective problems are plotted in Fig. 8 to Fig. 10, respectively.

Fig. 8 shows the six Pareto front approximations with three objectives. Clearly, the optimizer TDEA performs the best: the solutions are uniformly located over the whole Pareto front. $\mathrm{AR}+\mathrm{Grid}$ takes the second place, with its solutions distributed widely but not so uniformly as those of TDEA. The solutions obtained by NSGA-II and DMO are similar and distributed into many clusters on the Pareto front. The only difference between them is that the former seems to achieve a better result in terms of spread. The solutions of IBEA are of great regularity: most of them are located orderly on the boundary

\footnotetext{
${ }^{3}$ The archive set is also maintained with the same size if required.
}

of the Pareto front, but the rest of the region is populated sparsely. Due to the lack of a diversity maintenance method, the optimizer AR performs the worst among the selected optimizers, with the solutions concentrating around three scattered extreme points, i.e., $(1,0,0),(0,1,0)$, and $(0,0,1)$, of the Pareto front. Clearly, from the results in Fig. 8, it is clear that DCI is able to accurately reflect the relative distribution of Pareto front approximations: an approximation with a higher DCI value means that it performs better regarding the tradeoff between spread and uniformity.

The Pareto front approximations of the six optimizers for the six-objective DTLZ2 are plotted by parallel coordinates in Fig. 9. As seen in the figure, the distribution of these approximations is consistent with the DCI results. The solutions obtained by NSGA-II fail to approach the Pareto front. In this case, none of them are located into the grid environment set by Eqs. (2) and (3), and thus the DCI value is assigned zero. The solutions of AR reach the optimal front, but nearly converge into a point. Although DMO performs significantly better than AR, its solutions do not appear to be well distributed over the whole Pareto front: most of them are grouped into many clusters, and some regions are lack of solutions. The rest three optimizers IBEA, TDEA, and AR+Grid perform better in terms of diversity maintenance, thereby producing higher DCI values. More specifically, IBEA, similar to the tri-objective case, prefers to converge into the boundary of the Pareto front, and only several solutions are obtained in other parts of the front. AR+Grid tends to be the most successful in maintaining diversity: its solutions have a better coverage of the optimal front than those of the other five optimizers.

Concerning the ten-objective case shown in Fig. 10, the distribution of the approximations is generally similar to that for the six-objective case. An interesting difference is that IBEA fails to find the whole Pareto front of the problem. In this case, DMO achieves a higher DCI value than IBEA. In addition, AR+Grid seems to show more advantage over TDEA in terms of diversity, and thus the difference between their DCI values is larger.

The above experiments have demonstrated the effectiveness of DCI on the problems with a linear or concave Pareto front. We now further examine its effectiveness when working on problems with other Pareto geometries. The test problem DTLZ7 has a disconnect Pareto front consisting of $2^{m-1}$ regions with both convex and concave shapes (where $m$ denotes the number of objectives), and is used to test an algorithm's ability to maintain subpopulations in disconnected portions of the objective space.

Fig. 11 shows the Pareto front approximations obtained by one typical run of the six algorithms on the tri-objective DTLZ7. As can be seen from the figure, AR and DMO fail to find all the four Pareto optimal regions. The solutions of AR concentrate in the top region and the solutions of DMO are distributed in the top and left ones. Despite having found all the four regions, IBEA struggles to develop extensity, with most of the solutions located on the boundary of the regions. NSGA-II, TDEA, and AR+Grid perform similarly in terms of extensity, but differently in terms of uniformity. The solutions of TDEA have the best distribution uniformity, 


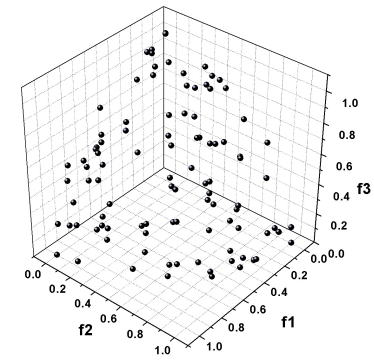

(a) NSGA-II (DCI $=0.5777)$

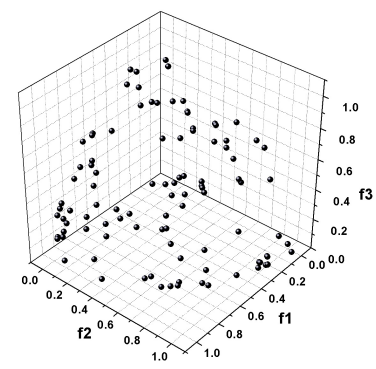

(d) DMO (DCI $=0.5455)$

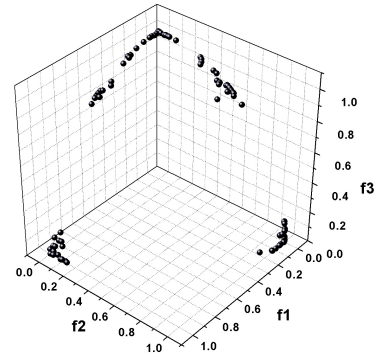

(b) $\mathrm{AR}(\mathrm{DCI}=0.1841)$

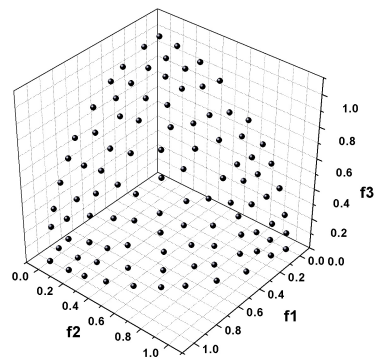

(e) TDEA (DCI $=0.7365)$

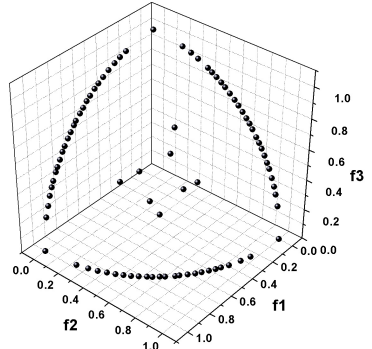

(c) $\operatorname{IBEA}(\mathrm{DCI}=0.3843)$

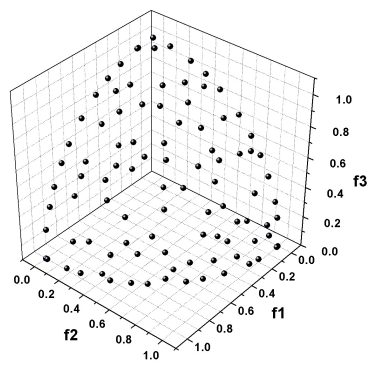

(f) $\mathrm{AR}+\mathrm{Grid}(\mathrm{DCI}=0.6927)$

Fig. 8. Pareto front approximations of the six algorithms and their DCI result on the tri-objective DTLZ2.

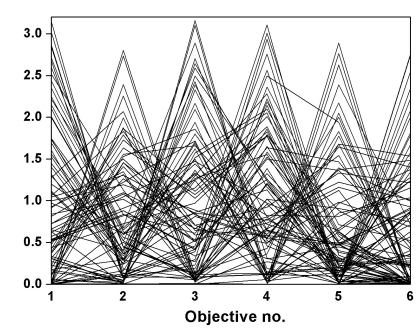

(a) NSGA-II $(\mathrm{DCI}=0)$

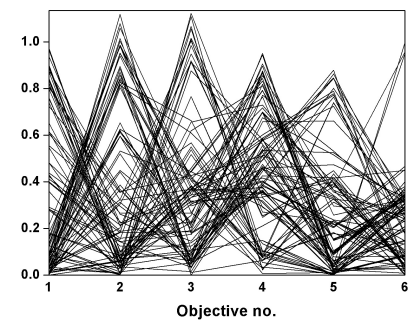

(d) $\mathrm{DMO}(\mathrm{DCI}=0.3942)$

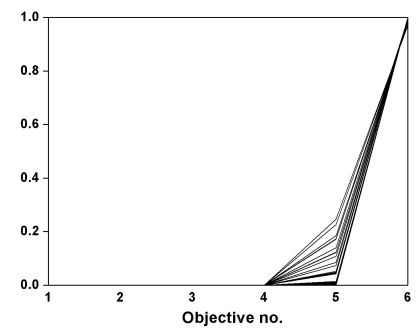

(b) $\mathrm{AR}(\mathrm{DCI}=0.0229)$

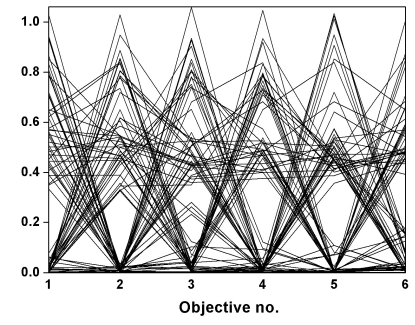

(e) TDEA (DCI $=0.5983)$

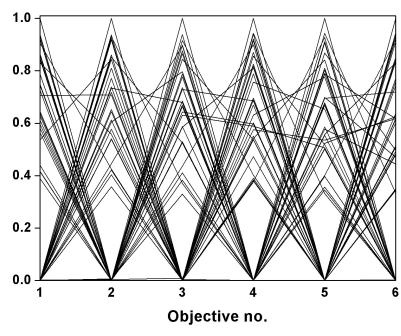

(c) $\operatorname{IBEA}(\mathrm{DCI}=0.5132)$

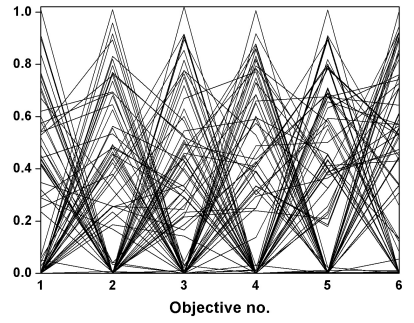

(f) $\mathrm{AR}+\mathrm{Grid}(\mathrm{DCI}=0.6391)$

Fig. 9. Pareto front approximations of the six algorithms and their DCI value on the six-objective DTLZ2 shown by parallel coordinates.

followed by those of AR+Grid and NSGA-II. Clearly, the assessment results of DCI confirm the above observations: an approximation with a higher DCI value means that it performs better regarding the comprehensive performance in finding multiple Pareto optimal regions as well as maintaining solutions' uniformity and extensity in each region.

\section{Comparative Study}

This section is devoted to comparing DCI with other diversity indicators. Here, two indicators, i.e., DM [30] and Maximum Spread (MS) [51], which are used widely in manyobjective optimization [32], [51], [66], [67], are chosen as the peers. DM, as briefly described before in Section II, is a gridbased indicator that can reflect the spread and uniformity of a Pareto front approximation. It takes the value in the range $[0,1]$. The larger the value, the better the diversity.

MS, originally presented in [49], measures the length of the diagonal of the hypercube formed by the extreme objective values in a Pareto front approximation. Since the original indicator can be influenced heavily by the convergence of the considered approximation, Adra and Fleming [51] improved the MS indicator by considering the extreme values of the Pareto front as follows:

$$
\operatorname{MS}(P)=\sqrt{\sum_{k=1}^{m}\left(\max _{p \in P}\left(p_{k}\right)-\min _{p \in P}\left(p_{k}\right)\right)^{2}} / \sqrt{\sum_{k=1}^{m}\left(n p_{k}-i p_{k}\right)^{2}}
$$

where $m$ denotes the number of objectives, $p_{k}$ denotes the 


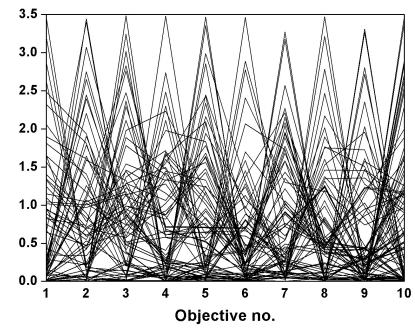

(a) NSGA-II (DCI =0)

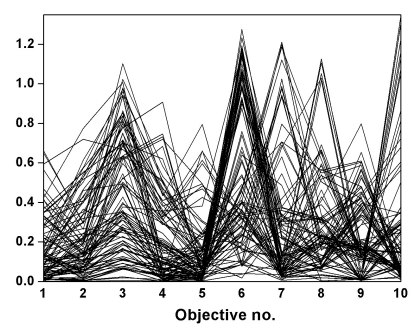

(d) DMO (DCI $=0.4266$ )

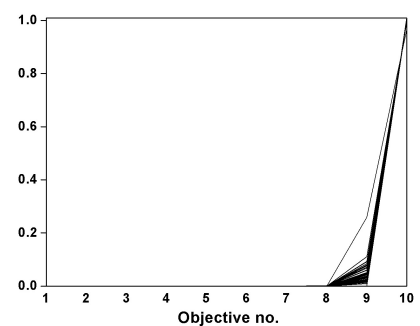

(b) $\mathrm{AR}(\mathrm{DCI}=0.0545)$

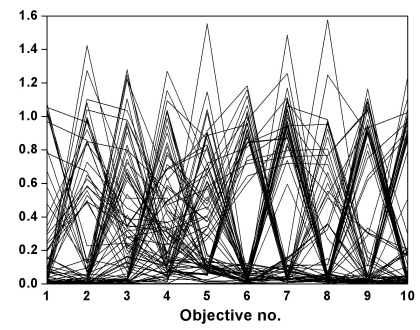

(e) TDEA (DCI $=0.4878$ )

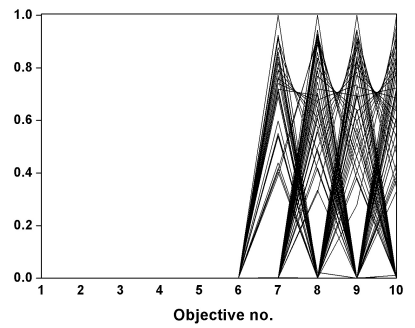

(c) $\operatorname{IBEA}(\mathrm{DCI}=0.3381)$

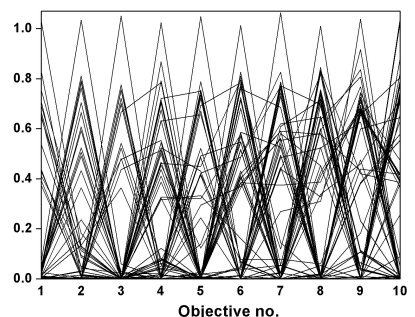

(f) $\mathrm{AR}+\mathrm{Grid}(\mathrm{DCI}=0.7590)$

Fig. 10. Pareto front approximations of the six algorithms and their DCI value on the ten-objective DTLZ2 shown by parallel coordinates.

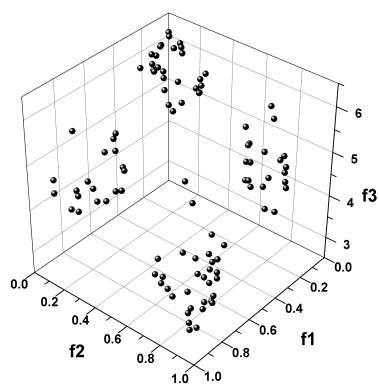

(a) NSGA-II (DCI $=0.6771)$

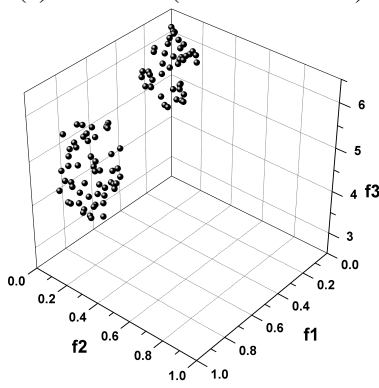

(d) DMO (DCI $=0.4513)$

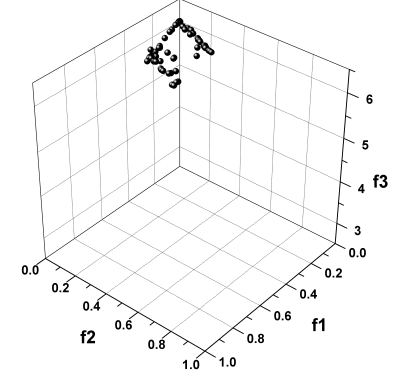

(b) $\mathrm{AR}(\mathrm{DCI}=0.1812)$

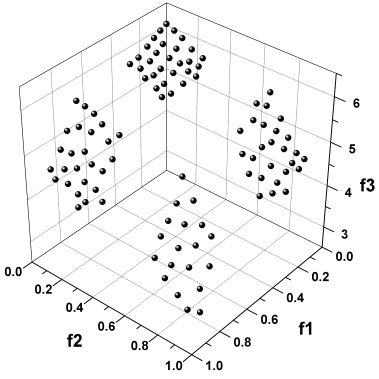

(e) TDEA (DCI $=0.8442)$

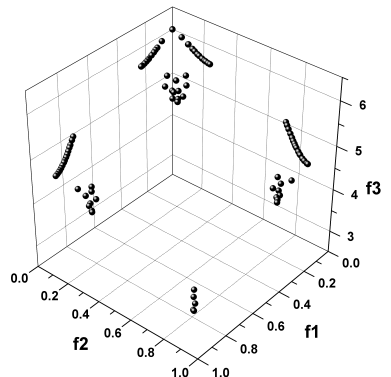

(c) $\operatorname{IBEA}(\mathrm{DCI}=0.4830)$

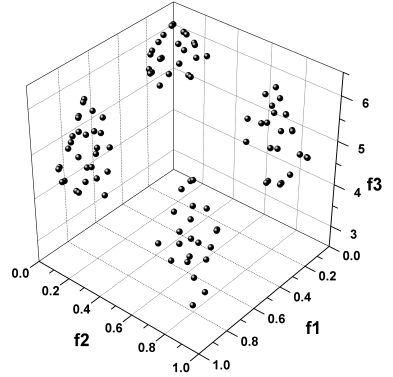

(f) $\mathrm{AR}+\mathrm{Grid}(\mathrm{DCI}=0.7673)$

Fig. 11. Pareto front approximations of the six algorithms and their DCI result on the tri-objective DTLZ7.

value of individual $p$ in the $k$ th objective, and $i p_{k}$ and $n p_{k}$ denote the value of the ideal point and Nadir point in the $k$ th objective, respectively.

The MS value of an approximation $P$ close to one (i.e., $\operatorname{MS}(P)=1$ ) is desired. The value smaller than one (i.e., $\operatorname{MS}(P)<1$ ) implies a lack of diversity of $P$ compared with the ideal result, which is most likely due to it converging into a sub-region of the Pareto front. The value larger than one (i.e., $\operatorname{MS}(P)>1$ ) indicates that $P$ is located far away from the Pareto front. In the light of the above properties, MS is used to not only compare the distribution range of approximations but also guide the search in many-objective optimization [51].

First, we compare DCI with DM. Here, we focus on the accuracy of the assessment result for the two indicators.
Therefore, their other differences (e.g., in DM each hyperbox in grid needs to be accessed and a reference set is also required; see items 27 and 28 in Table I) are not considered.

In DM, the setting of a hyperbox's neighborhood is crucial for the assessment result. Two hyperboxes are called as neighbors to each other if the Manhattan distance of their grid coordinates is not larger than one. However, this setting, when the number of objectives of a problem is large, may cause an inaccurate estimation of the position of individuals, further leading to an inaccurate assessment of approximations' diversity.

Let us consider a five-objective example with three hyperboxes $h_{1}(0,0,0,0,0), h_{2}(0,0,0,0,2)$, and $h_{3}(1,1,1,1,1)$. In $\mathrm{DM}, h_{3}$, rather than $h_{2}$, is a neighbor of $h_{1}$. Without loss 


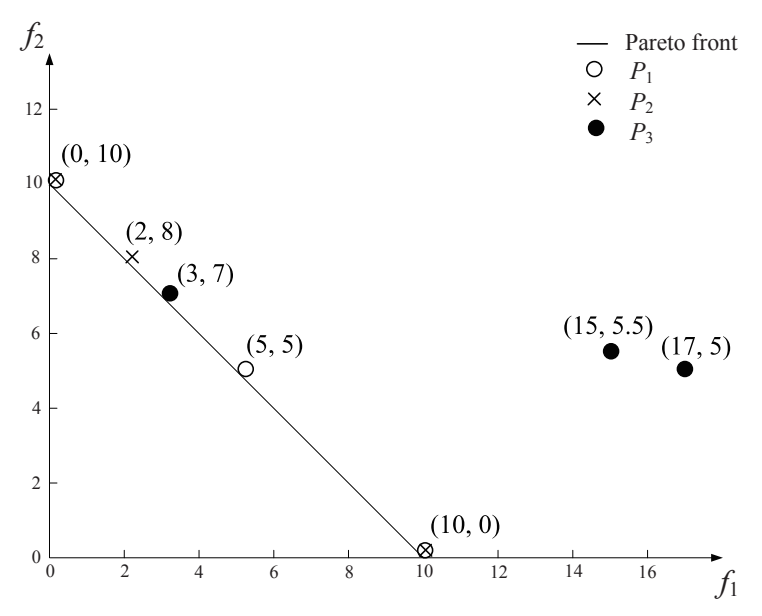

Fig. 12. An example of distribution of approximations for the MS and DCI indicators. $\operatorname{MS}\left(P_{1}\right)=\operatorname{MS}\left(P_{2}\right)=\operatorname{MS}\left(P_{3}\right)=1$, and $\operatorname{DCI}\left(P_{1}\right)=12 / 15>$ $\operatorname{DCI}\left(P_{2}\right)=11 / 15>\operatorname{DCI}\left(P_{3}\right)=7 / 15($ div $=5)$.

of generality, let individuals $\mathbf{A}, \mathbf{B}$, and $\mathbf{C}$ be located in the center of hyperboxes $h_{1}, h_{2}$, and $h_{3}$, respectively. Clearly, the Euclidean distance between $\mathbf{B}$ and $\mathbf{A}$ is shorter than that between $\mathbf{C}$ and $\mathbf{A}(2<\sqrt{5})$, but only individual $\mathbf{C}$ is in the neighborhood of $\mathbf{A}$.

Let there be two approximations $P$ and $Q$, with $P$ consisting of $\mathbf{A}$ and $\mathbf{B}$ and $Q$ consisting of $\mathbf{A}$ and $\mathbf{C}$. In this case, $\operatorname{DM}(P)>\operatorname{DM}(Q)$ because the distance between $\mathbf{A}$ and $\mathbf{B}$ is mistakenly considered farther than that between $\mathbf{A}$ and $\mathbf{C}$, in view of the location of $\mathbf{A}$ and $\mathbf{B}$ in different neighborhoods in DM. However, as to the proposed DCI indicator, the distance between individuals is calculated based on the Euclidean distance of their grid coordinates. Therefore, $\operatorname{DCI}(P)=\left(C D\left(P, h_{1}\right)+C D\left(P, h_{2}\right)+C D\left(P, h_{3}\right)\right) / 3=$ $(1+1+1 / 6) / 3=13 / 18<\operatorname{DCI}(Q)=\left(C D\left(Q, h_{1}\right)+\right.$ $\left.C D\left(Q, h_{2}\right)+C D\left(Q, h_{3}\right)\right) / 3=(1+1 / 3+1) / 3=14 / 18$.

Next, we consider the MS indicator. Since MS only tests the range comparison between an approximation and the problem's Pareto front, it may fail to assess the uniformity of solutions in the approximation. Moreover, even if testing the distribution range, MS may also give an inaccurate estimation because a poorly-converged approximation often has a broad range.

As an explanation to the problems of MS, Fig. 12 shows three approximations $P_{1}=\{(0,10),(5,5),(10,0)\}, P_{2}=$ $\{(0,10),(2,8),(10,0)\}$, and $P_{3}=\{(3,7),(15,5.5),(17,5)\}$ for a bi-objective optimization problem with a Pareto front $f_{1}+f_{2}=10$. Clearly, $P_{1}$ outperforms $P_{2}$ in terms of diversity, and two individuals in $P_{3}$ fail to approach the Pareto front. However, all the three approximations have the ideal MS result, i.e., $\operatorname{MS}\left(P_{1}\right)=\operatorname{MS}\left(P_{2}\right)=\operatorname{MS}\left(P_{3}\right)=1$. Concerning the DCI indicator, the two individuals in $P_{3}$ will be removed since they are dominated by some individuals in $P_{1}$ and $P_{2}$. The DCI results of three approximations are: $\operatorname{DCI}\left(P_{1}\right)=12 / 15>\operatorname{DCI}\left(P_{2}\right)=11 / 15>\operatorname{DCI}\left(P_{3}\right)=7 / 15$.

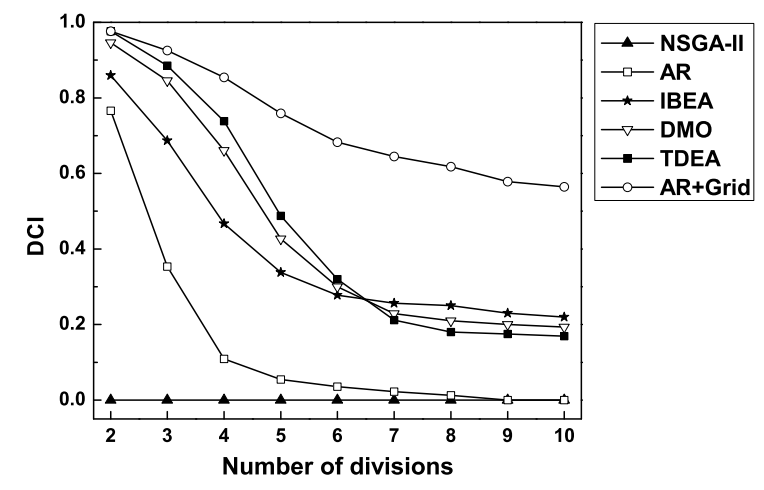

Fig. 13. DCI against the number of divisions of the six optimizers on the ten-objective DTLZ2.

TABLE V

DCI RESULTS OF THE SIX OPTIMIZERS ON THE 10-OBJECTIVE DTLZ2 WITH DIFFERENT NUMBERS OF DIVISIONS ( $d i v$ ). THE RESULTS ARE UNDERLINED WHEN THEY CAN CORRECTLY REFLECT THE DISTRIBUTION DIFFERENCES AMONG THE OPTIMIZERS

\begin{tabular}{c|c|c|c|c|c|c|c|c|c}
\hline div & 2 & 3 & 4 & 5 & 6 & 7 & 8 & 9 & 10 \\
\hline NSGA-II & 0 & $\mathbf{0}$ & $\mathbf{0}$ & $\mathbf{0}$ & 0 & 0 & 0 & 0 & 0 \\
AR & 0.766 & $\mathbf{0 . 3 5 3}$ & $\mathbf{0 . 1 0 9}$ & $\mathbf{0 . 0 5 5}$ & 0.036 & 0.023 & 0.012 & 0 & 0 \\
IBEA & 0.860 & $\mathbf{0 . 6 8 6}$ & $\mathbf{0 . 4 6 7}$ & $\mathbf{0 . 3 3 8}$ & 0.278 & 0.257 & 0.250 & 0.230 & 0.219 \\
DMO & 0.946 & $\mathbf{0 . 8 4 5}$ & $\mathbf{0 . 6 6 1}$ & $\mathbf{0 . 4 2 7}$ & 0.300 & 0.229 & 0.210 & 0.200 & 0.193 \\
TDEA & 0.977 & $\mathbf{0 . 8 8 5}$ & $\mathbf{0 . 7 3 9}$ & $\mathbf{0 . 4 8 8}$ & 0.320 & 0.212 & 0.180 & 0.175 & 0.169 \\
AR+Grid & 0.977 & $\mathbf{0 . 9 2 5}$ & $\mathbf{0 . 8 5 4}$ & $\mathbf{0 . 7 5 9}$ & 0.682 & 0.645 & 0.618 & 0.578 & 0.565 \\
\hline
\end{tabular}

\section{Study of Different Configurations of the Parameter div}

In DCI, a parameter div (the number of divisions) is required to divide the grid environment. Obviously, it largely affects the evaluation results since it determines the hyperbox location where Pareto front approximations are distributed. In this section, we investigate the effect of div and try to find an appropriate setting for a given problem. Here, we only show the results on a ten-objective problem due to the space limitation. Similar results can be obtained for problems with other numbers of objectives.

Fig. 13 plots the curves of the DCI results against the number of divisions for the six optimizers; they are also summarized in Table $\mathrm{V}$ for clarity. Clearly, there are two properties about the influence of div on the DCI. The first one is that the DCI value, in general, degrades with the growth of div. This is because the number of non-empty hyperboxes in grid increases with div, and for an approximation, more hyperboxes that are occupied by other approximations are needed to be considered. The second property is that within a certain range (div $\in[3,5]$ ), DCI can correctly compare the distributions of the approximations. Although the DCI values of the three competitive optimizers (i.e., IBEA, DMO, and TDEA) are all decreased, their differences remain mostly unchanged when div $\in[3,5]$. This provides an effective evaluation for the distributions of the approximations. On the other hand, when div is equal to 2, the DCI differences between TDEA and AR+Grid are too slight to reflect the distributions; and when div becomes larger than 6, the DCI values among IBEA, DMO, and TDEA may result in an incorrect judgment on their distributions. This occurrence may 
be attributed to the following reason: a very small div makes many solutions located in a hyperbox no matter how they are distributed; a large div causes that there are no other solutions existed in the neighborhood of a solution, which decreases the sensitivity of DCI to the extensity of approximations.

Intuitively, the optimal setting of div is the minimum required number of divisions of satisfying the condition that for a Pareto front approximation with ideal distribution (i.e., its individuals distributed uniformly over the whole Pareto front), the neighborhood ${ }^{4}$ of each individual does not contain any other individuals. This means that, ideally, the hyperboxes, in the dimensionality of the Pareto front of a given problem (i.e., the dimensionality of the manifold of Pareto optimal solutions in the objective space), should exactly cover all individuals so that no individual is located in the neighborhood of others. Namely,

$$
d i v^{n}=V_{(n, R)} \times N
$$

where $V_{(n, R)}$, representing the volume of a hyperbox's neighborhood in the dimensionality of the Pareto front, denotes the volume of an $n$-dimensional hypersphere with radius $R$, $n$ denotes the dimensionality of the Pareto front, $m$ denotes the number of objectives (obviously, $n \leq m-1$ ), and $N$ is the size of the approximation. Here, $R=\sqrt{m+1} / 2$, since two hyperboxes contribute nothing to each other (i.e, are nonneighboring) when their grid distance is larger than (or equal to) $\sqrt{m+1}$ (cf. Eq. (8)).

However, the optimal setting of div according to Eq. (10) may be infeasible in practice due to two reasons: 1) In DCI the neighborhood of a hyperbox corresponds to a hypersphere rather than a hypercube constructed by a set of hyperboxes, and 2) The shape of the Pareto front can be distinct for different problems, even if they have the same dimensionality.

Considering a bi-objective problem with one-dimensional manifold of the Pareto front, the closest grid distance between two non-neighboring individuals is $\sqrt{m+1}=\sqrt{2+1}=\sqrt{3}$. However, in the 2-dimensional objective space, there are not two hyperboxes whose grid distance is equal to $\sqrt{3}$ (the grid distance between two hyperboxes $\in\{1, \sqrt{2}, 2, \sqrt{5}, 2 \sqrt{2}, 3 \ldots\})$. In addition, approximations with different Pareto front shapes may require different numbers of hyperboxes. Fig. 14 gives a 2-objective example regarding two approximations, one $\left(P_{1}\right)$ with a straight line Pareto front and the other $\left(P_{2}\right)$ with a folding line Pareto front. Clearly, 49 hyperboxes can contain only 4 uniformly-distributed non-neighboring individuals from $P_{1}$ but 7 ones from $P_{2}$. This means that $P_{1}$ requires more hyperboxes than $P_{2}$ when they have the same number of individuals.

In view of these reasons, we give div a rough range estimation rather than a precise value. On the one hand, according to Eq. (10), div $=\left(V_{(n, \sqrt{m+1} / 2)} N\right)^{1 / n}>\left(V_{(n, \sqrt{m} / 2)} N\right)^{1 / n}$. On the other hand, as mentioned before, sometimes there are no two hyperboxes whose grid distance is equal to the ideal grid distance $(\sqrt{m+1})$. In this case, the radius of a hyperbox's neighborhood is larger than $\sqrt{m+1} / 2$. For instance, for a 2objective problem (e.g., the example in Fig. 14), the radius of

\footnotetext{
${ }^{4}$ Here, the neighborhood of an individual means the neighborhood of the hyperbox where the individual is located.
}

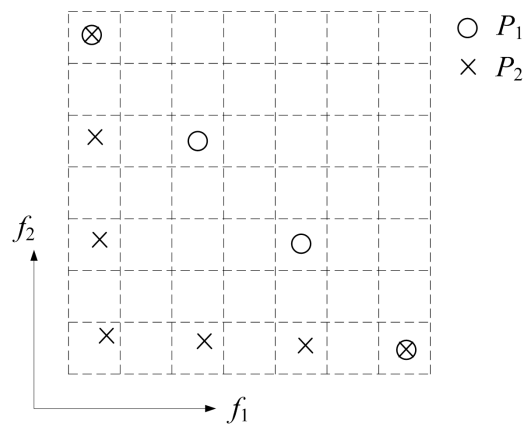

Fig. 14. An example of approximations with different Pareto front shapes requiring different numbers of hyperboxes.

the hyperbox's neighborhood will be $1, \sqrt{5} / 2$, or $\sqrt{2}$. In fact, under the condition of having the same number of individuals, an approximation with the $45^{\circ}$ hyperplane shape (like $P_{1}$ in Fig. 14) requires the most hyperboxes. In this case, the radius of the hyperbox's neighborhood will be equal to $\sqrt{m}$. Consequently, div $\leq\left(V_{(n, \sqrt{m})} N\right)^{1 / n}$.

Moreover, since div must be an integer and the total number of hyperboxes should be larger than (or equal to) that of hyperboxes occupied by individuals and their corresponding neighborhoods, therefore we have

$$
\left\lceil\left(V_{\left(n, R_{1}\right)} N\right)^{1 / n}\right\rceil \leq \operatorname{div} \leq\left\lceil\left(V_{\left(n, R_{2}\right)} N\right)^{1 / n}\right\rceil
$$

where $R_{1}=\sqrt{m} / 2$ and $R_{2}=\sqrt{m}$. For an $n$-dimensional hypersphere, its volume $V_{(n, R)}$ can be calculated by the following formula:

$$
V_{(n, R)}= \begin{cases}\left(\pi^{k} / k !\right) R^{n}, & \text { for } n=2 k \\ \left(2^{2 k+1} k ! \pi^{k} /(2 k+1) !\right) R^{n}, & \text { for } n=2 k+1\end{cases}
$$

where $k$ is an integer larger than or equal to zero. Taking the 4objective DTLZ2 as an example, here $n=3, R_{1}=1$ and $R_{2}=$ 2. So, $V_{(n, R)}=\frac{4}{3} \pi R^{3}$, div $\in\left[\left\lceil\left(\frac{4}{3} \pi N\right)^{1 / 3}\right\rceil,\left\lceil\left(\frac{32}{3} \pi N\right)^{1 / 3}\right\rceil\right]$, and further div is in the range $[8,15]$ if $N=100$.

Although the effectiveness of DCI seems to be insensitive to div in a certain range, different div settings will prefer different distributions of Pareto front approximations in the context of spread and uniformity. Next, we give two examples to explain this fact. Figs. 15 and 16 show two groups of Pareto front approximations obtained by $\mathrm{AR}+\mathrm{Grid}$ with different parameter settings on the 3- and 4-objective DTLZ2; the DCI results for different div settings are also included in the figures. Clearly, the solutions in Figs. 15(a) and 16(a) are located extensively but non-uniformly since they concentrate (or even coincide) in a number of scattered regions; whereas the solutions in Figs. 15(c) and 16(c) are uniformly distributed (i.e., adjacent solutions have basically equal spacing) but fail to cover the whole Pareto front.

An interesting observation from the comparison between $P_{1}$ and $P_{3}$ in the figures is that with a small $d i v$, an extensivelydistributed approximation has a better DCI value, while with a large div, a uniformly-distributed approximation is preferred. This phenomenon is mainly due to the sensitivity of DCI to the number of hyperboxes in grid. A small div makes hyperboxes decreased in number; an extensively-distributed approximation 


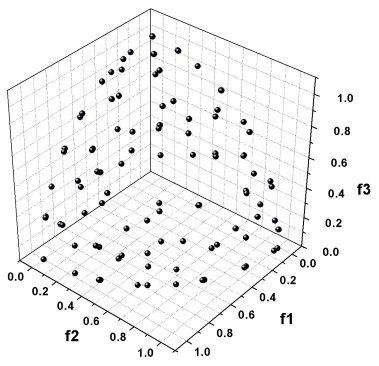

(a) $P_{1}(d=14)$

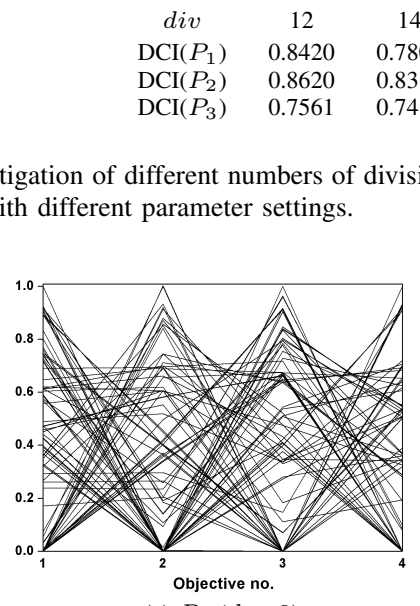

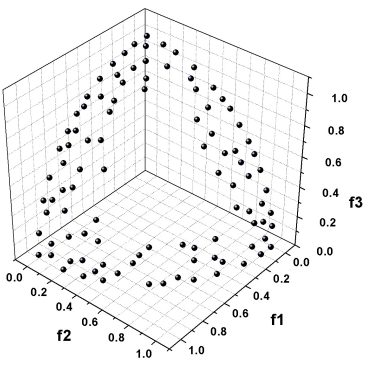

(c) $P_{3}(d=28)$

Fig. 15. Investigation of different numbers of divisions (div) in DCI for the tri-objective DTLZ2. The Pareto front approximations $P_{1}, P_{2}$, and $P_{3}$ obtained by AR+Grid with different parameter settings.

(a) $P_{1}(d=9)$

$\begin{array}{cc}\text { div } & 8 \\ \operatorname{DCI}\left(P_{1}\right) & 0.795 \\ \operatorname{DCI}\left(P_{2}\right) & 0.865 \\ \operatorname{DCI}\left(P_{3}\right) & 0.716\end{array}$

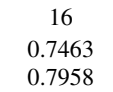

17
0.7353
0.7946

0.7946
0.6985

18
0.7188
0.7704
0.6967

19
0.6893
0.7535

20

0.6559

0.7369 21 0.6278 0.7059 $\begin{array}{cc}23 & 25 \\ 0.5887 & 0.5710 \\ 0.6860 & 0.6600\end{array}$ $0.6553 \quad 0.6335$

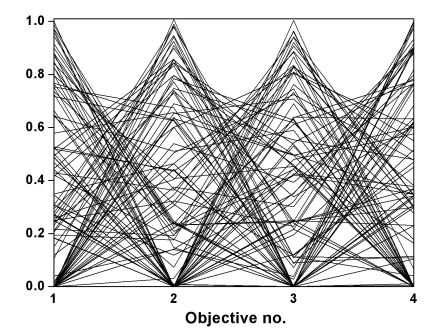

(b) $P_{2}(d=17)$

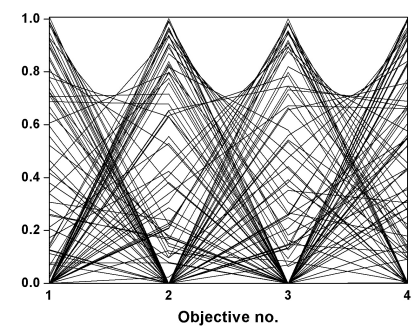

(c) $P_{3}(d=30)$

$\begin{array}{ccccccc}9 & 10 & 11 & 12 & 13 & 14 & 15 \\ 0.7255 & 0.6792 & 0.6360 & 0.5902 & 0.5398 & 0.5256 & 0.4833 \\ 0.8436 & 0.8203 & 0.7899 & 0.7625 & 0.7307 & 0.6812 & 0.6558 \\ 0.6649 & 0.6473 & 0.6350 & 0.6259 & 0.5991 & 0.5940 & 0.5858\end{array}$

Fig. 16. Investigation of different numbers of divisions (div) in DCI for the four-objective DTLZ2. The Pareto front approximations $P_{1}, P_{2}$, and $P_{3}$ obtained by AR+Grid with different parameter settings.

will cover (or at least be not far away from) any considered hyperbox, thereby obtaining a relatively higher average contribution degree. On the other hand, when div becomes larger, the number of hyperboxes increases, and correspondingly the grid distance between solutions becomes longer; the solutions that are uniformly distributed and are away from each other with certain distance will have a higher likelihood in different hyperbox neighborhoods, thereby providing relatively higher contributions to the evaluation result.

The above properties can make the setting of div in the DCI consistent with the preference of the user. In the absence of guidance information, the optimal setting, if attainable, is first suggested (e.g., div $=19$ for a 3-objective problem with a 2-dimensional manifold of Pareto front when the size of approximations is equal to 100); otherwise, a value around the median of the range in Eq. (11) can be replaced. On the other hand, a slightly lower (or higher) div is recommended if the user prefers an extensive (or uniform) distribution of the solutions in Pareto front approximations.

\section{E. Discussion}

None of performance indicators can assess all kinds of distributions of approximations in the area. Like existing diversity indicators, DCI also fails to deal with some specific distributions. Since DCI considers relative positions of two (several) approximations in a grid, it may not be able to distinguish approximations with similar (or same) relative positions in the grid but different distributions in the objective space. This is a weakness of the proposed indicator, which will be one important issue for our future study. A possible way to cope with this problem may be to assign different weights to the considered hyperboxes according to their distributions in the objective space.

In addition, DCI does not consider the difference between individuals in the same hyperbox. This can affect the accuracy of the assessment result to some extent. A refined division of grid (i.e., a large div) can reduce the probability of individuals located in same hyperboxes, but it decreases the sensitivity of DCI to the extensity of approximations, as explained in the previous section.

Here, it is worth mentioning that DCI only fails to assess several approximations which are located in the exactly same set of hyperboxes. In most cases, DCI can reflect the information regarding several approximations where there exist individuals distributed in the same hyperboxes. For example, considering two approximations $P$ and $Q$ with the same number of individuals, if $P$ has more individuals in the same hyperboxes than $Q$, in general $P$ will obtain a poorer DCI value since it provides fewer hyperboxes to be considered in the DCI calculation. 


\section{CONCLUSiOns}

This paper presents a quality indicator, DCI, to assess the diversity of Pareto front approximations in many-objective optimization. DCI can be used to compare the ability of different population-based multiobjective optimizers in generating well-distributed Pareto front approximations. In the implementation of DCI, all the concerned approximations are put into a grid environment and thus distributed in different hyperboxes. The proposed indicator considers the contribution of different approximations to the hyperboxes where the nondominated solutions in these approximations are located, thereby providing the following characteristics:

- DCI assesses the relative quality of different Pareto front approximations rather than provides an absolute measure of distribution for a single approximation. In other words, it delivers no information about the distribution of one approximation, but a quantitative comparison among several approximations in terms of diversity.

- DCI can identify any number of Pareto front approximations in a single run. In addition, it has a quadratic computational complexity, which is fully independent of the division setting in grid and does not increase with the number of hyperboxes.

- DCI does not require a reference set that substitutes the Pareto front of a given problem, which is especially suitable for many-objective optimization since an accurate substitution of a Pareto front is difficult or even impossible when the front's dimension is high.

- DCI is sensitive to the number of divisions in grid. Nonetheless, it works effectively within a certain range of division settings. Furthermore, the users can set the number of divisions according to their preference: a lower (or higher) division number can be set if they prefer extensive (or uniform) distribution of the solutions in Pareto front approximations.

Systematic experiments have been conducted by validating DCI on artificial and real Pareto front approximations with $3,4,5,6,8$, and 10 objectives. The evaluation results have confirmed the observations from the illustration. A comparison with two popular diversity indicators has been made to show the applicability of the proposed indicator in many-objective optimization. Moreover, the investigation of the effect of the parameter div on DCI has also been included in the experiments. Based on the experimental observations, some suggestions regarding how to set the parameter appropriately have been provided for the user.

Finally, it is worth mentioning that DCI may also be used to compare solution sets in preference-based multiobjective optimization, given that the range of the grid environment in the indicator can be determined by the user's interest.

\section{REFERENCES}

[1] J. Branke, K. Deb, K. Miettinen, and R. Slowinski, Multiobjective Optimization: Interactive and Evolutionary Approaches. Berlin, Heidelberg: Springer-Verlag, 2008.

[2] C. A. C. Coello, D. A. V. Veldhuizen, and G. B. Lamont, Evolutionary Algorithms for Solving Multi-Objective Problems, 2nd ed. Heidelberg: Springer, 2007.
[3] K. Deb, Multi-Objective Optimization Using Evolutionary Algorithms. New York: John Wiley, 2001.

[4] G. Leguizamón and C. A. C. Coello, "Multi-objective ant colony optimization: A taxonomy and review of approaches," Integration of Swarm Intelligence and Artificial Neural Network, pp. 67-94, 2011.

[5] H. Li and D. Landa-Silva, "An adaptive evolutionary multi-objective approach based on simulated annealing," Evol. Comput., vol. 19, no. 4, pp. 561-595, 2011.

[6] M. P. Hansen and A. Jaszkiewicz, "Evaluating the quality of approximations to the nondominated set," Institute of Mathematical Modeling, Technical University of Denmark, IMM-REP-1998-7, 1998.

[7] T. Okabe, Y. Jin, and B. Sendhoff, "A critical survey of performance indices for multi-objective optimisation," in Proc. IEEE Congr. Evol. Comput., vol. 2, 2003, pp. 878-885.

[8] J. D. Knowles, L. Thiele, and E. Zitzler, "A tutorial on the performance assessment of stochastic multiobjective optimizers," Computer Engineering and Networks Laboratory (TIK), ETH Zurich, Switzerland, Tech. Rep. No. 214, 2006.

[9] E. Zitzler, J. Knowles, and L. Thiele, "Quality assessment of Pareto set approximations," in Multiobjective Optimization, J. Branke, K. Deb, K. Miettinen, and R. Slowinski, Eds. Springer Berlin / Heidelberg, 2008, vol. 5252, pp. 373-404.

[10] G. G. Yen and Z. He, "Performance metrics ensemble for multiobjective evolutionary algorithms," IEEE Trans. Evol. Comput., vol. 18, no. 1, pp. 131-144, Feb. 2014.

[11] M. Farina and P. Amato, "Fuzzy optimality and evolutionary multiobjective optimization," in Evolutionary Multi-Criterion Optimization. Faro, Portugal: Springer Berlin / Heidelberg, 2003, pp. 58-72.

[12] P. Fleming, R. Purshouse, and R. Lygoe, "Many-objective optimization: An engineering design perspective," in Evolutionary Multi-Criterion Optimization. Springer Berlin / Heidelberg, 2005, pp. 14-32.

[13] H. Ishibuchi, N. Tsukamoto, and Y. Nojima, "Evolutionary manyobjective optimization: A short review," in Proc. IEEE Congr. Evol. Comput., 2008, pp. 2419-2426.

[14] D. W. Corne and J. D. Knowles, "Techniques for highly multiobjective optimisation: some nondominated points are better than others," in Proc. 9th Annual Conf. Genetic Evol. Comput., 2007, pp. 773-780.

[15] E. J. Hughes, "Fitness assignment methods for many-objective problems," in Multiobjective Problem Solving from Nature. Springer Berlin Heidelberg, 2008, pp. 307-329.

[16] M. Li, S. Yang, and X. Liu, "Shift-based density estimation for paretobased algorithms in many-objective optimization," IEEE Trans. Evol. Comput., 2014, in press.

[17] A. L. Jaimes, L. V. S. Quintero, and C. A. Coello Coello, "Ranking methods in many-objective evolutionary algorithms," in Nature-Inspired Algorithms for Optimisation, R. Chiong, Ed., 2009, pp. 413-434.

[18] X. Zou, Y. Chen, M. Liu, and L. Kang, "A new evolutionary algorithm for solving many-objective optimization problems," IEEE Trans. Syst., Man, Cybern. B, vol. 38, no. 5, pp. 1402-1412, 2008.

[19] S. Yang, M. Li, X. Liu, and J. Zheng, "A grid-based evolutionary algorithm for many-objective optimization," IEEE Trans. Evol. Comput., vol. 17, no. 5, pp. 721-736, Oct. 2013.

[20] K. Deb, L. Thiele, M. Laumanns, and E. Zitzler, "Scalable test problems for evolutionary multiobjective optimization," in Evolutionary Multiobjective Optimization. Theoretical Advances and Applications, A. Abraham, L. Jain, and R. Goldberg, Eds. Springer Berlin Heidelberg, 2005, pp. 105-145.

[21] H. Aguirre and K. Tanaka, "Working principles, behavior, and performance of MOEAs on MNK-landscapes," Eur. J. Oper. Res., vol. 181, no. 3, pp. 1670-1690, 2007.

[22] H. Ishibuchi, Y. Hitotsuyanagi, N. Tsukamoto, and Y. Nojima, "Manyobjective test problems to visually examine the behavior of multiobjective evolution in a decision space," in Parallel Problem Solving from Nature - PPSN XI. Springer Berlin / Heidelberg, 2010, pp. 91-100.

[23] D. Saxena, Q. Zhang, J. Duro, and A. Tiwari, "Framework for manyobjectivet test problems with both simple and complicated Pareto-set shapes," in Evolutionary Multi-Criterion Optimization. Springer Berlin / Heidelberg, 2011, pp. 197-211.

[24] H. Ishibuchi, N. Akedo, and Y. Nojima, "A many-objective test problem for visually examining diversity maintenance behavior in a decision space," in Proc. 13th Annual Conf. Genetic Evol. Comput., 2011, pp. 649-656.

[25] M. Li, S. Yang, and X. Liu, "A test problem for visual investigation of high-dimensional multi-objective search," in Proc. IEEE Congr. Evol. Comput., 2014, submitted. 
[26] A. L. Jaimes and C. A. Coello Coello, "Study of preference relations in many-objective optimization," in Proc. 11th Annual Conf. Genetic Evol. Comput., 2009, pp. 611-618.

[27] K. Deb and H. Jain, "An improved NSGA-II procedure for manyobjective optimization part I: solving problems with box constraints," KanGAL, Indian Institute of Technology, Tech. Rep. 2012009, 2012.

[28] M. Li, S. Yang, X. Liu, and R. Shen, "A comparative study on evolutionary algorithms for many-objective optimization," in Evolutionary Multi-Criterion Optimization, 2013, pp. 261-275.

[29] E. Zitzler and L. Thiele, "Multiobjective evolutionary algorithms: a comparative case study and the strength Pareto approach," IEEE Trans. Evol. Comput., vol. 3, no. 4, pp. 257-271, Aug. 1999.

[30] K. Deb and S. Jain, "Running performance metrics for evolutionary multi-objective optimization," KanGAL, Indian Institute of Technology, Tech. Rep. 2002004, 2002.

[31] J. Wu and S. Azarm, "Metrics for quality assessment of a multiobjective design optimization solution set," J. Mech. Des., vol. 123, pp. 18-25, 2001.

[32] V. Khare, X. Yao, and K. Deb, "Performance scaling of multi-objective evolutionary algorithms," in Evolutionary Multi-Criterion Optimization. Springer Berlin / Heidelberg, 2003, pp. 376-390.

[33] T. Wagner, N. Beume, and B. Naujoks, "Pareto-, aggregation-, and indicator-based methods in many-objective optimization," in Evolutionary Multi-Criterion Optimization. Springer Berlin / Heidelberg, 2007, pp. $742-756$.

[34] J. D. Knowles and D. W. Corne, "On metrics for comparing nondominated sets," in Proc. Congr. Evol. Comput., vol. 1, 2002, pp. 711-716.

[35] E. Zitzler, L. Thiele, M. Laumanns, C. M. Fonseca, and V. G. da Fonseca, "Performance assessment of multiobjective optimizers: an analysis and review," IEEE Trans. Evol. Comput., vol. 7, no. 2, pp. 117-132, Apr. 2003.

[36] C. Fonseca and P. Fleming, "On the performance assessment and comparison of stochastic multiobjective optimizers," in Parallel Problem Solving from Nature-PPSN IV, 1996, pp. 584-593.

[37] D. A. Van Veldhuizen and G. B. Lamont, "Evolutionary computation and convergence to a Pareto front," in Late Breaking Papers at the Genetic Programming Conference, 1998, pp. 221-228.

[38] O. Schütze, X. Esquivel, A. Lara, and C. A. C. Coello, "Using the averaged Hausdorff distance as a performance measure in evolutionary multi-objective optimization," IEEE Trans. Evol. Comput., vol. 16, no. 4, pp. 504-522, Aug. 2012.

[39] J. R. Schott, "Fault tolerant design using single and multicriteria genetic algorithm optimization," Master's thesis, Department of Aeronautics and Astronautics, Massachusetts Institute of Technology, 1995.

[40] D. A. Van Veldhuizen, "Multiobjective evolutionary algorithms: Classifications, analyses, and new innovations," Ph.D. dissertation, Faculty of the Graduate School of Engineering of the Air Force Institute of Technology, Air University, 1999.

[41] D. A. Van Veldhuizen and G. B. Lamont, "On measuring multiobjective evolutionary algorithm performance," in Proc. Congr. Evol. Comput., vol. 1, 2000, pp. 204-211.

[42] K. Tan, T. Lee, and E. Khor, "Evolutionary algorithms for multiobjective optimization: Performance assessments and comparisons," Artif. Intel. Rev., vol. 17, pp. 251-290, 2002.

[43] P. Czyzak and A. Jaszkiewicz, "Pareto simulated annealing-a metaheuristic technique for multiple-objective combinatorial optimization," J. of Multi-Criteria Decision Analysis, vol. 7, no. 1, pp. 34-47, 1998.

[44] E. Zitzler and L. Thiele, "Multiobjective optimization using evolutionary algorithms: a comparative case study," in Parallel Problem Solving from Nature - PPSN V. Springer Berlin / Heidelberg, 1998, pp. 292-301.

[45] S. Bandyopadhyay, S. Pal, and B. Aruna, "Multiobjective gas, quantitative indices, and pattern classification," IEEE Trans. Syst., Man, Cybern. B, vol. 34, no. 5, pp. 2088-2099, 2004.

[46] K. Deb, S. Agrawal, A. Pratap, and T. Meyarivan, "A fast elitist nondominated sorting genetic algorithm for multi-objective optimization: NSGA-II," in Parallel Problem Solving from Nature-PPSN VI. Springer Berlin / Heidelberg, 2000, pp. 849-858.

[47] A. Farhang-Mehr and S. Azarm, "An information-theoretic metric for assessing multi-objective optimization solution set quality," J. Mech. Des., vol. 125, no. 4, pp. 655-663, 2004.

[48] M. Li, J. Zheng, and G. Xiao, "Uniformity assessment for evolutionary multi-objective optimization," in Proc. IEEE Congr. Evol. Comput., 2008, pp. 625-632.

[49] E. Zitzler, K. Deb, and L. Thiele, "Comparison of multiobjective evolutionary algorithms: Empirical results," Evol. Comput., vol. 8, no. 2, pp. 173-195, Jun. 2000.
[50] C. K. Goh and K. C. Tan, "An investigation on noisy environments in evolutionary multiobjective optimization," IEEE Trans. Evol. Comput., vol. 11 , no. 3, pp. 354-381, Jun. 2007.

[51] S. F. Adra and P. J. Fleming, "Diversity management in evolutionary many-objective optimization," IEEE Trans. Evol. Comput., vol. 15, no. 2, pp. 183-195, Apr. 2011.

[52] M. Li and J. Zheng, "Spread assessment for evolutionary multi-objective optimization," in Evolutionary Multi-Criterion Optimization, Nantes, France, 2009, pp. 216-230.

[53] P. A. N. Bosman and D. Thierens, "The balance between proximity and diversity in multiobjective evolutionary algorithms," IEEE Trans. Evol. Comput., vol. 7, no. 2, pp. 174-188, 2003

[54] Q. Zhang, A. Zhou, and Y. Jin, "RM-MEDA: A regularity model-based multiobjective estimation of distribution algorithm," IEEE Trans. Evol. Comput., vol. 12, no. 1, pp. 41-63, Feb. 2008.

[55] E. Zitzler, "Evolutionary algorithms for multiobjective optimization: Methods and applications," Ph.D. dissertation, Zurich, Switzerland: Swiss Federal Institute of Technology (ETH), 1999.

[56] G. Lizárraga-Lizárraga, A. Hernández-Aguirre, and S. Botello-Rionda, "G-Metric: an M-ary quality indicator for the evaluation of nondominated sets," in Proc. 10th Annual Conf. Genetic Evol. Comput.,, 2008, pp. 665-672.

[57] G. Lizárraga-Lizárraga, "On the evaluation of the quality of nondominated sets," Ph.D. dissertation, Center for Research in Mathematics A.C., Computer Science Area, Guanajuato, Mexico, 2009.

[58] K. Deb, A. Pratap, S. Agarwal, and T. Meyarivan, "A fast and elitist multiobjective genetic algorithm: NSGA-II," IEEE Trans. Evol. Comput., vol. 6, no. 2, pp. 182-197, Apr. 2002

[59] S. Mostaghim and J. Teich, "A new approach on many objective diversity measurement," in Practical Approaches to Multi-Objective Optimization, 2005.

[60] M. Li, S. Yang, J. Zheng, and X. Liu, "ETEA: A Euclidean minimum spanning tree-based evolutionary algorithm for multiobjective optimization," Evol. Comput., vol. 22, no. 2, Summer 2014.

[61] N. Beume, B. Naujoks, and M. Emmerich, "SMS-EMOA: Multiobjective selection based on dominated hypervolume," Eur. J. Oper. Res., vol. 181, no. 3, pp. 1653-1669, 2007.

[62] Q. Zhang and H. Li, "MOEA/D: A multiobjective evolutionary algorithm based on decomposition," IEEE Trans. Evol. Comput., vol. 11, no. 6, pp. 712-731, Dec. 2007.

[63] J. Bader and E. Zitzler, "HypE: An algorithm for fast hypervolumebased many-objective optimization," Evol. Comput., vol. 19, no. 1, pp. 45-76, Jul. 2011.

[64] A. Auger, J. Bader, D. Brockhoff, and E. Zitzler, "Theory of the hypervolume indicator: Optimal $\mu$-distributions and the choice of the reference point," in Proceedings of the tenth ACM SIGEVO workshop on Foundations of genetic algorithms (FOGA 09), 2009, pp. 87-102.

[65] E. Zitzler, L. Thiele, and J. Bader, "On set-based multiobjective optimization," IEEE Trans. Evol. Comput., vol. 14, no. 1, pp. 58-79, Feb. 2010.

[66] F. di Pierro, S.-T. Khu, and D. A. Savić, "An investigation on preference order ranking scheme for multiobjective evolutionary optimization," IEEE Trans. Evol. Comput., vol. 11, no. 1, pp. 17-45, Feb. 2007.

[67] M. Li, J. Zheng, K. Li, Q. Yuan, and R. Shen, "Enhancing diversity for average ranking method in evolutionary many-objective optimization," in Parallel Problem Solving from Nature-PPSN XI, 2010, pp. 647-656.

[68] K. Miettinen, Nonlinear Multiobjective Optimization. Boston: Kluwer Academic Publishers, 1999.

[69] M. Ehrgott and D. Tenfelde-Podehl, "Computation of ideal and nadir values and implications for their use in MCDM methods," Eur. J. Oper. Res., vol. 151, no. 1, pp. 119-139, 2003

[70] P. J. Bentley and J. P. Wakefield, "Finding acceptable solutions in the Pareto-optimal range using multiobjective genetic algorithms," in Soft Computing in Engineering Design and Manufacturing, 1997, ch. 5, pp. 231-240.

[71] E. Zitzler and S. Künzli, "Indicator-based selection in multiobjective search," in Parallel Problem Solving from Nature-PPSN VIII. Springer Berlin / Heidelberg, 2004, pp. 832-842.

[72] I. Karahan and M. Köksalan, "A territory defining multiobjective evolutionary algorithm and preference incorporation," IEEE Trans. Evol. Comput., vol. 14, no. 4, pp. 636-664, Aug. 2010. 


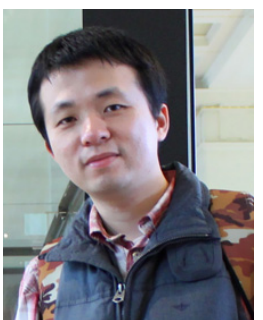

Miqing $\mathbf{L i}$ received the B.Sc. degree in computer science from the School of Computer and Communication, Hunan University, China, in 2004, and the M.Sc. degree in computer science from the College of Information Engineering, Xiangtan University, China, in 2008, respectively. He is currently pursuing the Ph.D. degree in the School of Information Systems, Computing, and Mathematics, Brunel University, U.K.

From 2008 to 2011, he was a Research Assistant in the College of Information Engineering, Xiangtan University, China. He has published over ten research papers since 2008. His current research interests include evolutionary computation, multiobjective optimization, and dynamic optimization.

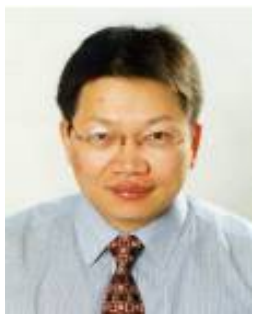

Shengxiang Yang (M'00) received the B.Sc. and M.Sc. degrees in automatic control and the Ph.D. degree in systems engineering from Northeastern University, Shenyang, China in 1993, 1996, and 1999, respectively.

$\mathrm{He}$ is currently a Professor in Computational Intelligence and Director of the Centre for Computational Intelligence, School of Computer Science and Informatics, De Montfort University, Leicester, U.K. He has over 170 publications. His current research interests include evolutionary and genetic algorithms, swarm intelligence, computational intelligence in dynamic and uncertain environments, artificial neural networks for scheduling, and relevant real-world applications.

Prof. Yang is the Chair of the Task Force on Evolutionary Computation in Dynamic and Uncertain Environments, Evolutionary Computation Technical Committee, IEEE Computational Intelligence Society, and the Founding Chair of the Task Force on Intelligent Network Systems, Intelligent Systems Applications Technical Committee, IEEE Computational Intelligence Society.

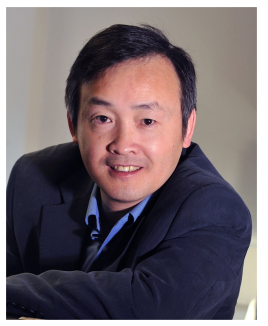

Xiaohui Liu is Professor of Computing at Brunel University in the UK where he directs the Centre for Intelligent Data Analysis, conducting interdisciplinary research concerned with the effective analysis of data. He is a Charted Engineer, Life Member of the Association for the Advancement of Artificial Intelligence, Fellow of the Royal Statistical Society, and Fellow of the British Computer Society. Professor Liu has over 100 high quality journal publications in biomedical informatics, computational intelligence, data mining and dynamic systems where he has more than 40 IEEE Transaction papers and his H-index is over 40. 